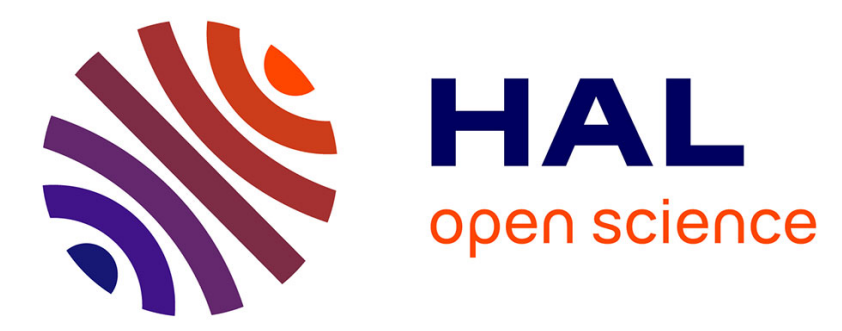

\title{
Designing Continuously Constrained Spacecraft Relative Trajectories for Proximity Operations
}

\author{
Georgia Deaconu, Christophe Louembet, Alain Théron
}

\section{To cite this version:}

Georgia Deaconu, Christophe Louembet, Alain Théron. Designing Continuously Constrained Spacecraft Relative Trajectories for Proximity Operations. Journal of Guidance, Control, and Dynamics, 2015, 38 (7), pp.1208-1217. 10.2514/1.G000283 . hal-01078528

\section{HAL Id: hal-01078528 \\ https://hal.science/hal-01078528}

Submitted on 29 Oct 2014

HAL is a multi-disciplinary open access archive for the deposit and dissemination of scientific research documents, whether they are published or not. The documents may come from teaching and research institutions in France or abroad, or from public or private research centers.
L'archive ouverte pluridisciplinaire HAL, est destinée au dépôt et à la diffusion de documents scientifiques de niveau recherche, publiés ou non, émanant des établissements d'enseignement et de recherche français ou étrangers, des laboratoires publics ou privés. 


\title{
using non negative polynomials
}

\author{
Georgia Deaconu ${ }^{1}$ and Christophe Louembet ${ }^{2}$ and Alain Théron ${ }^{3}$ \\ CNRS; LAAS; 7 avenue du colonel Roche, F-31400 Toulouse; France \\ Univ de Toulouse; UPS, LAAS; F-31400 Toulouse; France
}

The article presents a new method for designing an optimal plan of impulsive maneuvers for spacecraft rendezvous that accounts for the presence of continuous constraints on the relative trajectory. Impulsive control and continuous constraints are brought together through the parameterization of the spacecraft relative positions between two consecutive maneuvers. By using a variable change and a polynomial approximation of the integral term in the expressions of the relative positions, the continuous constraints on the trajectory can be transformed into non negativity constraints of some polynomials on a given interval. The resulting optimal control problem is solved using semi-definite programming.

\section{Introduction}

In the recent years, the needs and requirements of spacecraft on-orbit servicing missions have been thoroughly analysed [1]. This kind of operations demand flexible control algorithms, capable of handling the type of hard constraints associated to spacecraft proximity maneuvers. During close vicinity rendezvous operations, in addition to the input constraints usually considered, hard constraints on the trajectory must also be addressed [2-5]. If, for instance, the LIDAR sensor is used in the estimation of the spacecraft relative state, the limited field of view of the equipment

\footnotetext{
1 Methods and Algorithms in Control, LAAS-CNRS, gdeaconu@laas.fr

2 Methods and Algorithms in Control, LAAS-CNRS, christophe.louembet@laas.fr

3 Methods and Algorithms in Control, LAAS-CNRS, atheron@laas.fr
} 
must be accounted for. This usually translates in constricting the rendezvous trajectory to remain within the visibility cone of the sensor [6-8].

The ability of the spacecraft to hover inside a specified volume in a fuel optimal manner is also a major feature in the on-orbit servicing missions. Target monitoring introduces some hard constraints on the relative trajectory since it requires for the chaser spacecraft to remain in a specified zone defined in the target-centered frame. This problem has been recently studied by $[9,10]$ with the purpose of maximizing the time spent by the spacecraft in a specified cylindrical region. The hovering capabilities are also used during the ATV (Automated Transfer Vehicle) flights to the International Space Station. For these missions, the rendezvous trajectory consists of several way-points that the spacecraft must reach and where it must wait for the authorisation to proceed [11]. The spacecraft is in fact placed on a periodic parking orbit around the desired waiting point since it is more efficient from a fuel consumption point of view than maintaining a fixed position. In this paper, the hovering operation refers to periodic relative orbits exclusively since they allow the chaser spacecraft to remain inside the specified region on an infinite time horizon with no fuel consumption.

The previous examples have in common the necessity of imposing constraints on the spacecraft relative trajectory. They express the need for a generic algorithm that can provide rigorous solutions to the constrained optimal control problems that follow from these different guidance scenarios. An algorithm that can be used for the design of such constrained spacecraft relative trajectories is presented in this paper.

The spacecraft relative motion with respect to arbitrary elliptical orbits was investigated by Tschauner and Hempel in [12]. A transition matrix that enables the usage of the closed form solutions for the spacecraft relative motion was presented in [13]. Conventional methods use this tool and the discretization of the constraints in order to obtain a desired relative trajectory: the relative motion is propagated at specified instants where the trajectory constraints are explicitly checked [5]. The main advantage of these methods is that they transform the optimal control problem into a tractable program. However, they do not account for the behaviour of the obtained trajectory in between the discretization points and violations of the constraints might occur on these 
Precise parametric characterisation of the geometry of the spacecraft relative trajectories would enable the designer to choose only those that continuously satisfy the given set of guidance constraints. Works in this area focused mainly on the case of periodic relative motion. Periodic trajectories can be obtained by imposing the equality between the semi-major axes of the spacecraft orbits [14]. A periodicity condition valid for the Cartesian non-linear model of relative motion was proposed by Gurfill in [15]. However, the periodicity condition alone does not provide any insight with respect to the geometry of the obtained trajectory and further investigations are necessary. Analytical expressions for the the minimum and the maximum distance between two spacecraft on elliptical orbits are given in [16] as a function of the orbital elements of the satellites. An eighth degree trigonometric polynomial must be solved in order to obtain the true anomalies corresponding to the worst case extremal distances, rendering the method too complex and too conservative to be used within a guidance algorithm. A step forward into the study of the geometry of the spacecraft relative motion was achieved in [17] where the effects of the eccentricity on the shape of the periodic trajectories are analyzed by means of a parametrization of the relative motion. A similar parametrization is presented in [18] in order to show that when satellites on elliptical orbits are considered the relative periodic trajectories are usually three-dimensional and then compute the number of self-intersections. In [19], the periodic trajectory is expressed as a function of the difference between the spacecraft orbital elements but the extremal separation distances are analytically calculated only for some particular cases. A constraints-discretization based method is used in [20] in order to obtain periodic relative trajectories that respect the imposed dimension constraints. The results present the same benefits and drawbacks inherent to this types of methods that were previously mentioned.

It becomes clear that the geometry of spacecraft relative periodic orbits can be studied using different kinds of parametric representations. While some interesting conclusions can be drawn, these parametrizations still lack a clear link between the parameters and the dimensions of the resulting trajectories in the general case. We propose a parametric representation for the spacecraft relative position that is based on the Yamanaka-Ankersen transition matrix [13] and we show how it can be 
used for designing constrained relative trajectories. The vector of parameters is directly linked to the initial conditions of the relative motion of the satellites through a linear function and the periodic motion can be treated as a particular case by requiring one of the parameters to be zero. In our approach, the linear constraints imposed on the spacecraft relative trajectory are translated into a linear relation between the proposed vector of parameters and the cone of the positive semi-definite matrices. The obtained solution is guaranteed to satisfy the trajectory constraints continuously in time. This represents a major improvement over the conventional discretization based methods which, for a similar algorithmic complexity, require a specific a posteriori checkout procedure in order to validate the solution. Furthermore, in the case of periodic relative motion, the proposed approach provides an analytical description of the set of states belonging to periodic trajectories that respect a given set of linear constraints.

The article is organized as follows. Section II presents the parametrization for the spacecraft relative trajectory, with the highlight on the link between the vector of parameters and the main design parameter, the initial spacecraft relative state. Rational expressions are given for the relative motion by using an appropriate variable change and a polynomial approximation of the drifting term $J$ of the Yamanaka-Ankersen transition matrix. In section III the spacecraft rendezvous guidance problem is stated as an optimal control problem with impulsive input under saturation constraints and under continuous state constraints. By using the rational expressions for the relative motion, the constraints on the state are translated into non negativity constraints on some polynomials. Results on non negative polynomials are then used to formulate the guidance problem as a semidefinite program. In section IV, several examples demonstrate the large range of rendezvous guidance problems that can be addressed using the proposed method.

\section{Parameterization of the spacecraft relative motion}

Consider two spacecraft on arbitrary elliptic Keplerian orbits, one spacecraft called the leader and the other called the follower or the chaser. The spacecraft are depicted as $M_{L}$ and $M_{F}$ respectively in figure 1 . The true anomaly $\nu$ expresses at each moment the position of the leader on its

orbit and it is measured in the perifocal basis $(\vec{P}, \vec{Q}, \vec{W})$. The relative dynamics are projected in a 
rotating Cartesian local-vertical/local-horizontal (LVLH) basis attached to the leader, $\left(\vec{x}_{L}, \vec{y}_{L}, \vec{z}_{L}\right)$

in figure 1 .

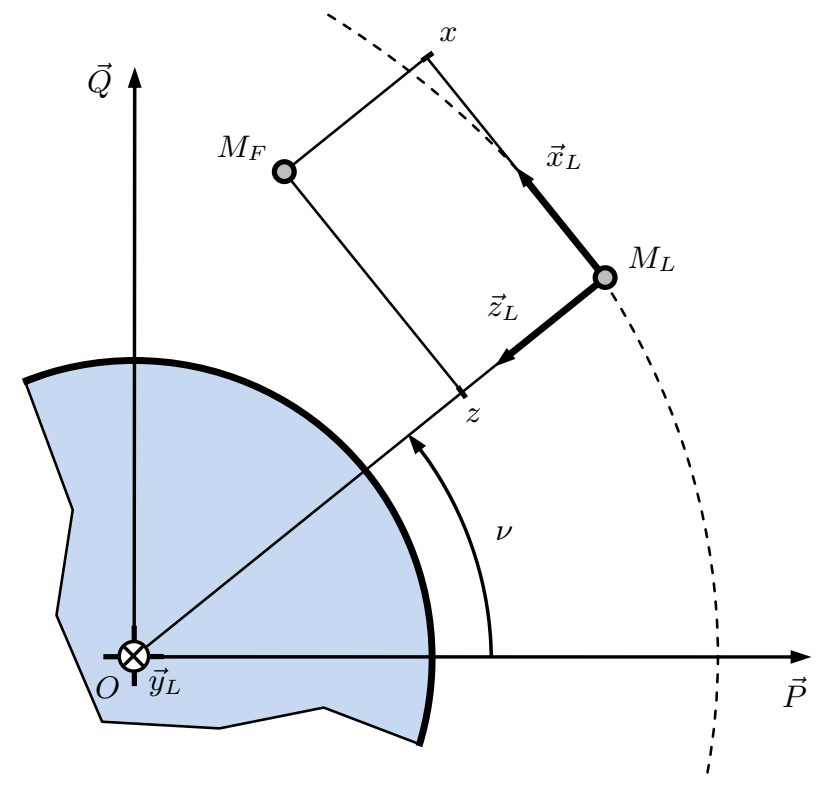

Fig. 1: The local LVLH frame attached to the leader and the spacecraft relative position

We assume that the distance between the leader and the follower spacecraft is much smaller than the distance between the leader and the center of the Earth. In this case, the linearized spacecraft relative motion expressed in the leader's LVLH frame can be described using the wellknown Tschauner-Hempel equations:

$$
\begin{aligned}
& \ddot{x}=2 \dot{\nu} \dot{z}+\ddot{\nu} z+\dot{\nu}^{2} x-\frac{\mu}{R^{3}} x+u_{x} \\
& \ddot{y}=-\frac{\mu}{R^{3}} y+u_{y} \\
& \ddot{z}=-2 \dot{\nu} \dot{x}-\ddot{\nu} x+\dot{\nu}^{2} z+2 \frac{\mu}{R^{3}} z+u_{z}
\end{aligned}
$$

where $\mu$ is the gravitational constant of the Earth,

$$
\dot{\nu}=\sqrt{\frac{\mu}{a^{3}\left(1-e^{2}\right)^{3}}}(1+e \cos \nu)^{2}, \quad R=\frac{a\left(1-e^{2}\right)}{1+e \cos \nu}
$$

and $a$ and $e$ are the semi-major axis and the eccentricity of the leader spacecraft. To obtain a simplified form of equations (1), the independent variable can be changed from the time to the leader's true anomaly $\nu$ through:

$$
\frac{d(.)}{d t}=\frac{d(.)}{d \nu} \frac{d \nu}{d t}=(.)^{\prime} \dot{\nu}
$$


and the following variable scaling can be introduced:

$$
\left[\begin{array}{c}
\tilde{x} \\
\tilde{y} \\
\tilde{z}
\end{array}\right]=(1+e \cos \nu)\left[\begin{array}{l}
x \\
y \\
z
\end{array}\right],\left[\begin{array}{l}
\tilde{x}^{\prime} \\
\tilde{y}^{\prime} \\
\tilde{z}^{\prime}
\end{array}\right]=-e \sin \nu\left[\begin{array}{l}
x \\
y \\
z
\end{array}\right]+\frac{1+e \cos \nu}{\dot{\nu}}\left[\begin{array}{l}
\dot{x} \\
\dot{y} \\
\dot{z}
\end{array}\right]
$$

This leads to the following equations for the relative motion:

$$
\begin{aligned}
& \tilde{x}^{\prime \prime}=2 \tilde{z}^{\prime}+\tilde{u}_{x} \\
& \tilde{y}^{\prime \prime}=-\tilde{y}+\tilde{u}_{y} \\
& \tilde{z}^{\prime \prime}=\frac{3}{1+e \cos \nu} \tilde{z}-2 \tilde{x}^{\prime}+\tilde{u}_{z}
\end{aligned}
$$

Starting from equations (5), Yamanaka and Ankersen [13] presented a transition matrix that can be used for the propagation of the relative motion starting from an initial state $\tilde{X}\left(\nu_{0}\right)$, under impulsive control $\Delta \tilde{V}$ :

$$
\tilde{X}(\nu)=\Phi_{\nu_{0}}^{\nu} \tilde{X}\left(\nu_{0}\right)+\sum_{i} \Phi_{\nu_{i}}^{\nu} \tilde{B} \Delta \tilde{V}_{i}
$$

where $\tilde{X}(\nu)=\left[\tilde{x}(\nu) \tilde{y}(\nu) \tilde{z}(\nu) \tilde{x}^{\prime}(\nu) \tilde{y}^{\prime}(\nu) \tilde{z}^{\prime}(\nu)\right]^{T}$. The matrix $\tilde{B}$ is given by $\tilde{B}=\left[\begin{array}{ll}0_{3} & I_{3}\end{array}\right]^{T}$, since the effect of the impulsive control is modelled as an instantaneous change in the relative speed.

Based on the definition of the transition matrix given in [13], a parametrization for the spacecraft relative positions is presented next. This parametrization is then used for designing impulsive maneuvers leading to spacecraft relative trajectories that respect continuously in time different types of linear constraints that are usually associated to spacecraft proximity operations.

\section{A. Non periodic spacecraft relative motion}

The evolution of the spacecraft relative trajectory between two consecutive impulsive controls, applied at $\nu_{0}$ and $\nu_{f}$ respectively, can be seen as the open loop propagation of the relative motion starting from the state right after that the first impulse is fired:

$$
\tilde{X}(\nu)=\Phi_{\nu_{0}}^{\nu}\left(\tilde{X}\left(\nu_{0}\right)+B \Delta \tilde{V}_{0}\right)=\Phi_{\nu_{0}}^{\nu} \tilde{X}^{+}\left(\nu_{0}\right), \quad \nu \in\left[\nu_{0}, \nu_{f}\right]
$$


Starting from the definition given in [13] for the transition matrix $\Phi$, the parametric equations for the autonomous relative trajectory are given by:

$$
\begin{aligned}
& \tilde{x}(\nu)=(2+e \cos \nu)\left(d_{1} \sin \nu-d_{2} \cos \nu\right)+d_{3}+3 d_{4} J(\nu)(1+e \cos \nu)^{2} \\
& \tilde{y}(\nu)=d_{5} \cos \nu+d_{6} \sin \nu \\
& \tilde{z}(\nu)=(1+e \cos \nu)\left(d_{2} \sin \nu+d_{1} \cos \nu\right)-3 e d_{4} J(\nu) \sin \nu(1+e \cos \nu)+2 d_{4}
\end{aligned}
$$

where the independent variable $\nu$ belongs to $\left[\nu_{0}, \nu_{f}\right]$ and the vector of parameters $D=$ $\left[\begin{array}{llllll}d_{1} & d_{2} & d_{3} & d_{4} & d_{5} & d_{6}\end{array}\right]^{T}$ depends linearly on $\tilde{X}_{0}=\tilde{X}^{+}\left(\nu_{0}\right)$, the relative state from which the satellites motion is propagated:

$$
D=C\left(\nu_{0}\right) \tilde{X}_{0}
$$

where:

$$
C(\nu)=\left[\begin{array}{cccccc}
0 & 0 & \frac{3(e+\cos \nu)}{e^{2}-1} & \frac{-\left(2 \cos \nu+e \cos ^{2} \nu+e\right)}{e^{2}-1} & 0 & \frac{\sin \nu(1+e \cos \nu)}{e^{2}-1} \\
0 & 0 & \frac{3 \sin \nu\left(1+e \cos \nu+e^{2}\right)}{\left(e^{2}-1\right)(1+e \cos \nu)} & \frac{-\sin \nu(2+e \cos \nu)}{e^{2}-1} & 0 & \frac{-\left(\cos \nu+e \cos ^{2} \nu-2 e\right)}{e^{2}-1} \\
1 & 0 & \frac{-3 e \sin \nu(2+e \cos \nu)}{\left(e^{2}-1\right)(1+e \cos \nu)} & \frac{e \sin \nu(2+e \cos \nu)}{e^{2}-1} & 0 & \frac{e^{2} \cos ^{2} \nu+e \cos \nu-2}{e^{2}-1} \\
0 & 0 & \frac{-\left(3 e \cos \nu+e^{2}+2\right)}{e^{2}-1} & \frac{(1+e \cos \nu)^{2}}{e^{2}-1} & 0 & \frac{-e \sin \nu(1+e \cos \nu)}{e^{2}-1} \\
0 & \cos \nu & 0 & 0 & -\sin \nu & 0 \\
0 & \sin \nu & 0 & 0 & \cos \nu & 0
\end{array}\right]
$$

The relative motion on the $y$ axis is naturally periodic, while the motion in the orbital plane is defined by a combination between periodic trigonometric terms and a drifting term denoted $J(\nu)$. This latter grows linearly in time and expressed as a function of the independent variable $\nu$ is given by:

$$
J(\nu)=\int_{\nu_{0}}^{\nu} \frac{d \tau}{(1+e \cos \tau)^{2}}=\frac{n\left(t-t_{0}\right)}{\left(1-e^{2}\right)^{3 / 2}}
$$

The following variable change can be used in order to remove the trigonometric terms in (8):

$$
w=\tan \left(\frac{\nu}{2}\right) \cos \nu=\frac{1-w^{2}}{1+w^{2}} \sin \nu=\frac{2 w}{1+w^{2}}
$$


Thus the parametric equations of the relative motion become:

$$
\begin{aligned}
\tilde{x}(w) & =\frac{1}{\left(1+w^{2}\right)^{2}}\left[P_{x}(w)+3 d_{4} P_{J x}(w) J(w)\right] \\
\tilde{y}(w) & =\frac{1}{1+w^{2}} P_{y}(w) \\
\tilde{z}(w) & =\frac{1}{\left(1+w^{2}\right)^{2}}\left[P_{z}(w)+2 d_{4} P_{J z}(w) J(w)\right]
\end{aligned}
$$

where $w \in\left[w_{0}, w_{f}\right]$ and:

$$
\begin{gathered}
J(w)=\int_{w_{0}}^{w} \frac{2 \tau^{2}+2}{\left((1-e) \tau^{2}+e+1\right)^{2}} d \tau \\
P_{x}(w)=\sum_{i=0}^{4} p_{x i} w^{i}, P_{y}(w)=\sum_{i=0}^{2} p_{y i} w^{i}, P_{z}(w)=\sum_{i=0}^{4} p_{z i} w^{i}, \\
P_{J x}(w)=\left((1+e)+(1-e) w^{2}\right)^{2}, \quad P_{J z}(w)=-3 e\left((1+e)+(1-e) w^{2}\right) w
\end{gathered}
$$

The coefficients $p_{x}=\left[\begin{array}{lllll}p_{x 0} & p_{x 1} & p_{x 2} & p_{x 3} & p_{x 4}\end{array}\right]^{T}, p_{y}=\left[\begin{array}{lll}p_{y 0} & p_{y 1} & p_{y 2}\end{array}\right]^{T}$ and $p_{z}=\left[\begin{array}{lllll}p_{z 0} & p_{z 1} & p_{z 2} & p_{z 3} & p_{z 4}\end{array}\right]^{T}$ of the polynomials $P_{x}(w), P_{y}(w)$ and $P_{z}(w)$ respectively depend linearly on the vector of parameters $D:$

$$
p_{x}=C_{x} D p_{y}=C_{y} D p_{z}=C_{z} D
$$

with:

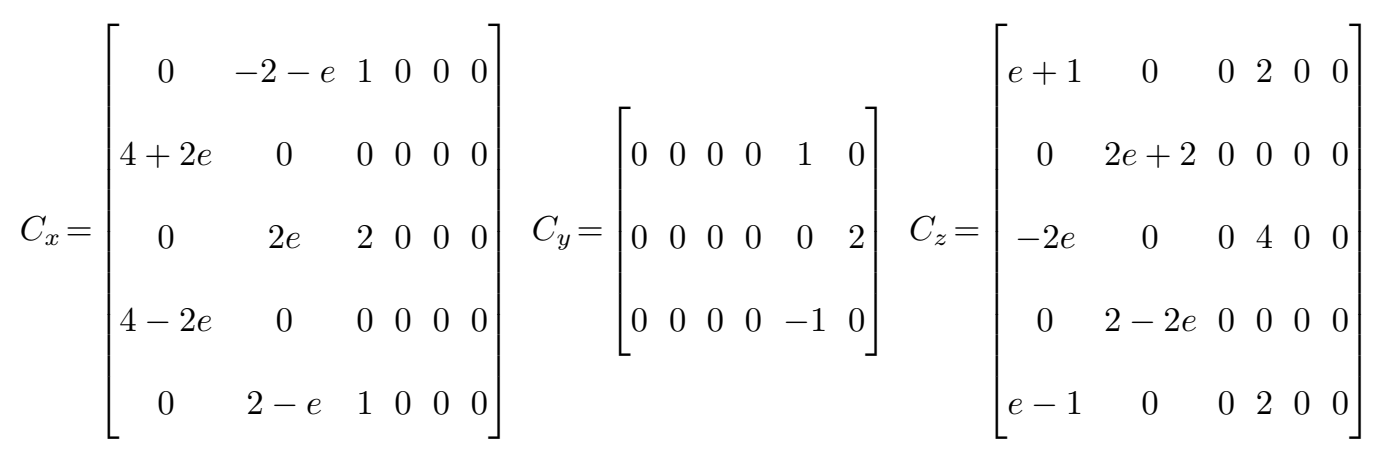

Hence the coefficients of these polynomials depend linearly on $\tilde{X}_{0}$, the relative state starting from which the satellites motion is propagated.

The presence of the drifting term $J$ causes the spacecraft relative trajectory to have an irrational form, regardless of whether the variable $\nu$ or the variable $w$ is used. Analytical rational expressions for the relative motion are instead needed in order to impose continuous constraints on the relative 
trajectory. They could allow the extension of the technique we developed in [21] to the case of non periodic relative motion. One way of obtaining the necessary rational expressions is presented next and it is based on using a polynomial approximation of the term $J$.

\section{B. Polynomial approximations for the drifting term $J(w)$}

The closed form expression of the integral (14) is given by:

$$
J(w)=\left[\frac{2 e w}{\left(e^{2}-1\right)\left((1-e) w^{2}+e+1\right)}-\frac{2 \operatorname{arctanh}\left(\frac{\sqrt{e-1}}{\sqrt{e+1}} w\right)}{\left(e^{2}-1\right)^{\frac{3}{2}}}\right]_{w_{0}}^{w}
$$

$\Theta_{u}(w)$ respectively, such that: It must be noted that the interval on which $J(w)$ may be approximated by a polynomial must be a finite subset of $\mathbb{R}$. In fact, $J(w)$ is discontinuous on the bounds of its definition set i.e. $\lim _{w \rightarrow-\infty} J(w) \neq \lim _{w \rightarrow+\infty} J(w)$ since $\lim _{t \rightarrow-1} \operatorname{arctanh}(t) \neq \lim _{t \rightarrow+1} \operatorname{arctanh}(t)$. Consequently, no polynomial or rational function can approximate $J(w)$ on $\mathbb{R}$. If the term $J(w)$ is replaced in (13) by a polynomial approximation $\Theta_{r}(w)$, valid on a finite interval $W=\left[w_{0}, w_{f}\right]$, then the expressions describing the spacecraft relative trajectory become rational:

$$
\begin{array}{ll}
\tilde{x}(w) & =\frac{1}{\left(1+w^{2}\right)^{2}}\left[P_{x}(w)+3 d_{4} P_{J x}(w) \Theta_{r}(w)\right] \\
\tilde{y}(w) & =\frac{1}{1+w^{2}} P_{y}(w)
\end{array} \quad, \forall w \in\left[w_{0}, w_{f}\right]
$$

Recent results from [22] show that a fixed-degree polynomial approximation $\Theta_{r}(w)$ with a certified maximum error can be obtained for the term $J(w)$ :

$$
J(w)=\Theta_{r}(w)+\varepsilon(w)
$$

where $\Theta_{r}$ is of degree $r$ and for $w_{0}=\tan \frac{\nu_{0}}{2}$ we have:

$$
\Theta_{r}\left(w_{0}\right)=J\left(w_{0}\right)=0
$$

The certified maximum error $\bar{\varepsilon}$ provided by the algorithm in [22] $\bar{\varepsilon}=\max _{w \in W} \varepsilon(w)$ is used in order to obtain upper and lower polynomial bounds on the term $J(\nu)$ :

$$
\Theta_{l}(w) \leq J(w) \leq \Theta_{u}(w), \forall w \in W=\left[w_{0}, w_{f}\right]
$$




$$
\Theta_{l}(w)=\Theta_{r}(w)-\bar{\varepsilon} \quad \Theta_{u}(w)=\Theta_{r}(w)+\bar{\varepsilon}
$$

\section{Periodic spacecraft relative motion}

From expressions (8) it can be noticed that the influence of the non periodic term $J(w)$ on the relative trajectory depends on the value of the parameter $d_{4}$. A periodic relative trajectory may be obtained by requiring the parameter $d_{4}$ to be zero and thus removing the drifting term. Imposing $d_{4}=0$ in (9) leads to a linear periodicity constraint on the relative state starting from which the spacecraft motion is propagated:

$$
M\left(\nu_{0}\right) X_{0}=0
$$

where the matrix $M(\nu) \in \mathbb{R}^{1 \times 6}$ is defined by:

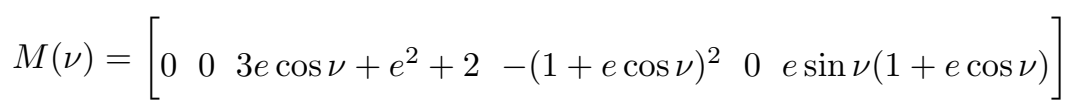

Assuming that the initial state $\tilde{X}_{0}$ satisfies the constraint (23), the trigonometric expressions for the propagation of the periodic trajectory are given by:

$$
\begin{array}{ll}
\tilde{x}(\nu)=(2+e \cos \nu)\left(d_{1}^{p} \sin \nu-d_{2}^{p} \cos \nu\right)+d_{3}^{p} & \\
\tilde{y}(\nu)=d_{5}^{p} \cos \nu+d_{6}^{p} \sin \nu & , \quad \forall \nu \geq \nu_{0} \\
\tilde{z}(\nu)=(1+e \cos \nu)\left(d_{2}^{p} \sin \nu+d_{1}^{p} \cos \nu\right) &
\end{array}
$$

where the vector of parameters $D_{p}=\left[\begin{array}{lllll}d_{1}^{p} & d_{2}^{p} & d_{3}^{p} & d_{5}^{p} & d_{6}^{p}\end{array}\right]^{T}$ is obtained from the substitution in (9) of the periodicity condition (23):

$$
D_{p}=C_{p}\left(\nu_{0}\right) \tilde{X}_{0}
$$

and:

$$
C_{p}(\nu)=\left[\begin{array}{cccccc}
0 & 0 & \frac{2 e \cos ^{2} \nu-e+\cos \nu}{(1+e \cos \nu)^{2}} & 0 & 0 & \frac{-\sin \nu}{1+e \cos \nu} \\
0 & 0 & \frac{\sin \nu(1+2 e \cos \nu)}{(1+e \cos \nu)^{2}} & 0 & 0 & \frac{\cos \nu}{1+e \cos \nu} \\
1 & 0 & \frac{e \sin \nu(2+e \cos \nu)}{(1+e \cos \nu)^{2}} & 0 & 0 & \frac{2+e \cos \nu}{1+e \cos \nu} \\
0 & \cos \nu & 0 & 0 & -\sin \nu & 0 \\
0 & \sin \nu & 0 & 0 & \cos \nu & 0
\end{array}\right]
$$


Introducing the variable change (12) in equations (25) leads to very similar expressions with respect

to the non periodic case. As expected, these expressions no longer contain the term $J(w)$ :

$$
\tilde{x}(w)=\frac{1}{\left(1+w^{2}\right)^{2}} P_{x p}(w), \tilde{y}(w)=\frac{1}{1+w^{2}} P_{y}(w), \quad \tilde{z}(w)=\frac{1}{\left(1+w^{2}\right)^{2}} P_{z p}(w) .
$$

The coefficients of the polynomials $P_{x p}(w)$ and $P_{z p}(w)$ depend linearly on the initial state of the propagation of the periodic motion through the vector of parameters $D_{p}$ :

$$
p_{x p}=C_{x} D_{p} \quad p_{z p}=C_{z} D_{p}
$$

where matrices $C_{x}$ and $C_{z}$ are defined in (18).

It is important to notice that in the periodic case the expressions for the relative positions are purely rational. Approximations are no longer required since the drifting term $J(w)$ has been removed. Expressions (28) are the same as those presented in [21] and we showed here that they are just a particular case of a more general parametrization of the spacecraft relative trajectory.

\section{Constrained spacecraft relative trajectory design}

The previous section showed that the spacecraft relative motion between two impulsive controls can be parametrized with respect to the state immediately after the first thrust: $\tilde{X}^{+}\left(\nu_{0}\right)=\tilde{X}\left(\nu_{0}\right)+$ $\tilde{B} \Delta \tilde{V}_{0}$. Thus the choice of $\Delta \tilde{V}_{0}$ plays a crucial role in obtaining a trajectory that continuously satisfies a specified set of constraints on the interval between the two consecutive controls. A new method for calculating the impulsive control $\Delta \tilde{V}_{0}$ leading to admissible trajectories is presented. The method is based on the previously developed parametrization for the relative motion. Let us assume that the constraints on the spacecraft relative path can be written in the general form of linear inequalities:

$$
V\left[\begin{array}{l}
\tilde{x}(\nu) \\
\tilde{y}(\nu) \\
\tilde{z}(\nu)
\end{array}\right] \leq \tilde{K}, \forall \nu \in\left[\nu_{0}, \nu_{f}\right]
$$

where $V \in \mathbb{R}^{s \times 3}, \tilde{K} \in \mathbb{R}^{s}, s$ is the number of constraints and $\tilde{K}=(1+e \cos \nu) K$ since the usage of the variable change (4) must be taken into account when writing the constraints. 
By using expressions (13) and the variable change (12), constraints (30) can be written as:

$$
v_{i, 1} \tilde{x}(w)+v_{i, 2} \tilde{y}(w)+v_{i, 3} \tilde{z}(w) \leq \frac{1+e+(1-e) w^{2}}{1+w^{2}} k_{i}, \forall w \in\left[w_{0}, w_{f}\right], i=1 . . s
$$

Let us define the rational expressions $\Xi_{i}(w)$ as:

$$
\Xi_{i}(w)=-v_{i, 1} \tilde{x}(w)-v_{i, 2} \tilde{y}(w)-v_{i, 3} \tilde{z}(w)+\frac{1+e+(1-e) w^{2}}{1+w^{2}} k_{i}=\frac{1}{\left(1+w^{2}\right)^{2}} \Gamma_{i}(w), i=1 . . s
$$

where:

$\Gamma_{i}(w)=-v_{i, 1}\left[P_{x}(w)+3 d_{4} P_{J x}(w) J(w)\right]-v_{i, 2} \bar{P}_{y}(w)-v_{i, 3}\left[P_{z}(w)+2 d_{4} P_{J z}(w) J(w)\right]+k_{i} T(w)$

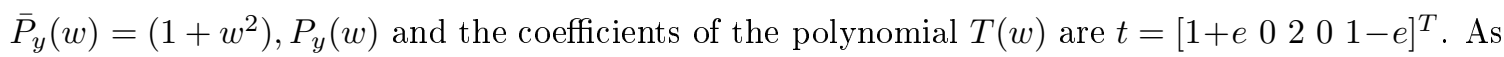
previously shown, the coefficients of the polynomials $P_{x}(w), \bar{P}_{y}(w)$ and $P_{z}(w)$ depend linearly on $\tilde{X}^{+}\left(\nu_{0}\right)$, the relative state from which the satellites motion is propagated:

$$
p_{x}=C_{x} C\left(\nu_{0}\right)\left(\tilde{X}\left(\nu_{0}\right)+\tilde{B} \Delta \tilde{V}_{0}\right) \bar{p}_{y}=\bar{C}_{y} C\left(\nu_{0}\right)\left(\tilde{X}\left(\nu_{0}\right)+\tilde{B} \Delta \tilde{V}_{0}\right) p_{z}=C_{z} C\left(\nu_{0}\right)\left(\tilde{X}\left(\nu_{0}\right)+\tilde{B} \Delta \tilde{V}_{0}\right)
$$

Hence, expressions $\Gamma_{i}(w)$ depend linearly on the decision variable $\Delta \tilde{V}_{0}$. Constraints (30) on the relative trajectory are satisfied if there exists an impulsive control $\Delta \tilde{V}_{0}$ such that:

$$
\exists \Delta \tilde{V}_{0} \quad \text { s.t. } \quad \Xi_{i}(w) \geq 0, \forall w \in\left[w_{0}, w_{f}\right], \forall i=1 . . s
$$

Since the common denominator for $\Xi_{i}(w)$ is $\left(1+w^{2}\right)^{2}$ which is non negative for all $w \in \mathbb{R}$, finding $\Delta \tilde{V}_{0}$ such that the expressions $\Gamma_{i}(w)$ are non negative on the given interval guarantees that the constraints (30) are respected:

$$
\exists \Delta \tilde{V}_{0} \quad \text { s.t. } \quad \Gamma_{i}(w) \geq 0, \forall w \in\left[w_{0}, w_{f}\right], \forall i=1 . . s
$$

It has been evidenced in the previous section the fact that the expressions $\Gamma_{i}(w)$ are irrational functions. The usage of the polynomial approximations for the term $J(\nu)$ allows constraints (36) to become polynomial non negativity constraints which can be reformulated as linear matrix inequality constraints [23]. These latter can be solved efficiently using convex programming methods. The uncertainties resulting from the approximation process can be directly accounted for. expressions $\Gamma_{i}(w)$ contain the integral term $J(w)$, they can be transformed into polynomial expressions by replacing $J(w)$ with a polynomial approximation. 


\section{A. The non-periodic case: using the polynomial approximation of the drifting term $J(w)$}

Expressions (33) can be transformed into polynomials by using the approximation (20). This introduces an unknown but bounded error $\varepsilon(w)$ such that:

$$
\Gamma_{i} \equiv \Gamma_{i}\left(w, \Theta_{r}(w), \varepsilon(w)\right)
$$

Thus the satisfaction of the uncertain constraints:

$$
\left.\exists \Delta \tilde{V}_{0} \quad \text { s.t. } \quad \Gamma_{i}\left(w, \Theta_{r}(w), \varepsilon(w)\right)\right) \geq 0, \forall \varepsilon(w) \in[-\bar{\varepsilon} \quad \bar{\varepsilon}], w \in\left[w_{0}, w_{f}\right], i=1 . . s
$$

is a sufficient condition for the satisfaction of constraints (36). Results from convex robust analysis [24] provide a robust counterpart to (37):

$$
\exists \Delta \tilde{V}_{0} \quad \text { s.t. } \quad\left(\begin{array}{c}
\Gamma_{i}^{l}\left(w, \Theta_{l}(w)\right) \geq 0 \\
\Gamma_{i}^{u}\left(w, \Theta_{u}(w)\right) \geq 0
\end{array}\right), w \in\left[w_{0}, w_{f}\right], i=1 . . s
$$

where $\Gamma_{i}^{u}\left(w, \Theta_{u}(w)\right)$ and $\Gamma_{i}^{l}\left(w, \Theta_{l}(w)\right)$ are the polynomials obtained after replacing the term $J(w)$ in (33) with its certified upper and lower polynomial bounds, $\Theta_{u}(w)$ and $\Theta_{l}(w)$ respectively (22).Therefore finding an impulsive control $\Delta \tilde{V}_{0}$ such that polynomials $\Gamma_{i}^{u}(w)$ and $\Gamma_{i}^{l}(w)$ are non negative guarantees that initial constraints (36) are satisfied, although solving this problem introduces some conservatism.

Reference [23] provides necessary and sufficient LMI conditions to check whether the coefficients of an univariate polynomial belong to the cone of coefficients of polynomials that are non negative on a finite interval. Since the coefficients $\gamma_{i}^{l}$ and $\gamma_{i}^{u}$ of the polynomials $\Gamma_{i}^{l}(w)$ and $\Gamma_{i}^{u}(w)$ depend linearly on $\Delta \tilde{V}_{0}$, these conditions can be used to find a suitable $\Delta \tilde{V}_{0}$ such that the constraints (38) on the propagated trajectory are satisfied.

Theorem 1 (Non negative polynomial on finite interval).

Let $\mathcal{K}_{a, b}$ be the convex, closed and pointed cone of polynomials that are non negative on a finite interval $[a, b] \in \mathbb{R}:$

$$
\mathcal{K}_{a, b}=\left\{p \in \mathbb{R}^{n+1}, P(w)=\sum_{i=0}^{n} p_{i} w^{i} \geq 0, \forall w \in[a, b]\right\}
$$

A polynomial $P(w)$, represented through its vector of coefficients $p=\left[p_{0}, \ldots, p_{n}\right]^{T}$, belongs to $\mathcal{K}_{a, b}$ if and only if there exist two symmetric positive semi-definite matrices $Y_{1}$ and $Y_{2}$ such that:

$$
p=\Lambda^{*}\left(Y_{1}, Y_{2}\right)
$$


Using this result, designing a relative trajectory for which the infinitely many constraints (30) are satisfied becomes equivalent to finding an impulsive control $\Delta \tilde{V}_{0}$ such that:

$$
\begin{aligned}
& \exists\left(Y_{i 1}^{l} \succeq 0, Y_{i 2}^{l} \succeq 0\right) \quad \text { s.t. } \quad \gamma_{i}^{l}=\Lambda^{*}\left(Y_{i 1}^{l}, Y_{i 2}^{l}\right) \\
& , i=1 . . s \\
& \exists\left(Y_{i 1}^{u} \succeq 0, Y_{i 2}^{u} \succeq 0\right) \quad \text { s.t. } \quad \gamma_{i}^{u}=\Lambda^{*}\left(Y_{i 1}^{u}, Y_{i 2}^{u}\right)
\end{aligned}
$$

The definition of the operator $\Lambda^{*}$ depends on whether the polynomial $P(w)$ has an odd or an even degree. For $n$ odd, take $m=(n-1) / 2$ and $Y_{1}, Y_{2} \in \mathbb{R}^{(m+1) \times(m+1)} \succeq 0$. Let $H_{k, i} \in \mathbb{R}^{(k+1) \times(k+1)}$ be some Henkel matrices that contain ones on the i-th anti-diagonal and zeros everywhere else. Then the operator $\Lambda^{*}$ is defined by:

$$
\Lambda^{*}\left(Y_{1}, Y_{2}\right)=\left[\begin{array}{c}
\operatorname{tr}\left(Y_{1}\left(-a H_{m, 1}\right)\right)+\operatorname{tr}\left(Y_{2}\left(b H_{m, 1}\right)\right) \\
\operatorname{tr}\left(Y_{1}\left(H_{m, 1}-a H_{m, 2}\right)\right)+\operatorname{tr}\left(Y_{2}\left(b H_{m, 2}-H_{m, 1}\right)\right) \\
\operatorname{tr}\left(Y_{1}\left(H_{m, i-1}-a H_{m, i}\right)\right)+\operatorname{tr}\left(Y_{2}\left(b H_{m, i}-H_{m, i-1}\right)\right) \\
\vdots \\
\operatorname{tr}\left(Y_{1} H_{m, 2 m+1}\right)+\operatorname{tr}\left(Y_{2}\left(-H_{m, 2 m+1}\right)\right)
\end{array}\right]
$$

For $n$ even, take $m=n / 2$ and $Y_{1} \in \mathbb{R}^{(m+1) \times(m+1)} \succeq 0, Y_{2} \in \mathbb{R}^{m \times m} \succeq 0$. Then the operator $\Lambda^{*}$ is defined by:

$$
\Lambda^{*}\left(Y_{1}, Y_{2}\right)=\left[\begin{array}{c}
\operatorname{tr}\left(Y_{1} H_{m, 1}\right)+\operatorname{tr}\left(Y_{2}\left(-a b H_{m-1,1}\right)\right) \\
\operatorname{tr}\left(Y_{1} H_{m, 2}\right)+\operatorname{tr}\left(Y_{2}\left((b+a) H_{m-1,1}-a b H_{m-1,2}\right)\right) \\
\operatorname{tr}\left(Y_{1} H_{m, 3}\right)+\operatorname{tr}\left(Y_{2}\left((b+a) H_{m-1,2}-H_{m-1,1}-a b H_{m-1,3}\right)\right) \\
\vdots \\
\operatorname{tr}\left(Y_{1} H_{m, i}\right)+\operatorname{tr}\left(Y_{2}\left((b+a) H_{m-1, i-1}-H_{m-1, i-2}-a b H_{m-1, i}\right)\right) \\
\vdots \\
\operatorname{tr}\left(Y_{1} H_{m, 2 m}\right)+\operatorname{tr}\left(Y_{2}\left((b+a) H_{m-1,2 m-1}-H_{m-1,2 m-2}\right)\right) \\
\operatorname{tr}\left(Y_{1}, H_{m, 2 m+1}\right)+\operatorname{tr}\left(Y_{2}\left(-H_{m-1,2 m-1}\right)\right)
\end{array}\right]
$$




\section{B. The periodic case}

When constraints (30) must be imposed on a periodic relative trajectory, expressions $\Gamma_{i}(w)$ are directly polynomial and no approximation is needed:

$$
\Gamma_{i}(w)=-v_{i, 1} P_{x p}(w)-v_{i, 2} \bar{P}_{y}(w)-v_{i, 3} P_{z p}(w)+k_{i} T(w) \geq 0, i=1 . . s
$$

Their coefficients can be written directly as a linear function of the decision variable $\Delta \tilde{V}_{0}$ :

$$
\gamma_{i}=\left(-v_{i, 1} C_{x}-v_{i, 2} \bar{C}_{y}-v_{i, 3} C_{z}\right) C_{p}\left(\nu_{0}\right)\left(\tilde{X}\left(\nu_{0}\right)+B \Delta \tilde{V}_{0}\right)+k_{i} t
$$

Note that in order for the expressions (43) to be valid the initial state for the propagation of the relative motion must satisfy the periodicity condition:

$$
M\left(\nu_{0}\right)\left(\tilde{X}\left(\nu_{0}\right)+B \Delta \tilde{V}_{0}\right)=0
$$

If constraints must be imposed on the periodic trajectory for an entire period, one must take into account the fact that the variable change (12) maps the trigonometric circle to $\mathbb{R}$, an infinite interval. The necessary and sufficient LMI conditions for non negativity of univariate polynomials on infinite intervals are slightly different with respect to the case where finite intervals are considered. Reference [23] demonstrates that a polynomial $P(w)$ is non negative on $\mathbb{R}$ if and only if there exists a symmetric positive semi-definite matrix $Y \in \mathbb{R}^{(m+1) \times(m+1)}$ such that the coefficients of the polynomial $P(w)$ verify:

$$
p=\Lambda^{*}(Y)
$$

where:

$$
\Lambda^{*}(Y)(j)=\operatorname{tr}\left(Y H_{m, j}\right), j=1 . .2 m+1
$$

It is interesting to notice that the polynomial non negativity constraints (43), along with the periodicity constraint (45), describe the set of spacecraft relative states that at $\nu_{0}$ belong to periodic trajectories that evolve inside the polytope defined by (30). This set represents an invariant set for the spacecraft relative motion [25]. The presented method allows the design of impulsive maneuvers leading to spacecraft relative trajectories that respect a set of linear constraints continuously in time. It is based on the parameterization of the spacecraft relative motion and makes use of 
the link between the cone of coefficients of non negative polynomials and the cone of symmetric positive definite matrices. The technique can easily be extended to accommodate a larger number of impulsive controls $\Delta \tilde{V}$ over a larger time horizon. This aspect is detailed in the applications section.

\section{Applications}

The method developed in the previous section can be used to design impulsive maneuvers for constrained spacecraft relative motion. In this section, we present three types of space missions where our approach can be effective. The hovering mission [9] requires the design of periodic trajectories that evolve inside a specified region. This type of trajectory should enable the visual inspection of a given target with an infinite time horizon for the observation task and with zero fuel cost. The passively safe rendezvous mission $[7,26]$ requests rendezvous trajectories that guarantee passive collision avoidance in case of anomalous system behaviour. Lastly, for the visibility constrained rendezvous mission $[4,7]$ visibility cone constraints must be satisfied all along the rendezvous path. If the first two scenarios belong to the periodic motion framework, for the rendezvous under visibility constraints scenario the constraints are continuously imposed on the non periodic relative motion. In each of these scenarios, it is assumed that the number of impulses $N$, the thrusting positions $\nu_{i}$ and the initial relative state $\tilde{X}_{1}$ are known.

\section{A. Generating a hovering trajectory}

The term hovering refers to the ability of a deputy spacecraft to remain in a specified area close to the target satellite [9], in order to inspect or to monitor it. The design of fuel efficient maneuvers leading to proximity naturally periodic relative trajectories between two satellites is an important aspect of the on-orbit inspection and on-orbit servicing missions. Moreover, this objective must to be achieved while taking into account the necessity of restricting the evolution of the resulting periodic trajectory to a specified region of the space. Starting from an initial state $\tilde{X}_{1}$, a final state $\tilde{X}\left(\nu_{N}\right)$ must be reached, state that respects the periodicity condition and guarantees that the resulting periodic trajectory remains inside a given tolerance region $R_{t o l}$. This must be done while minimizing the fuel cost necessary to attain this final state and respecting the saturation constraints 
on the amplitude of the thrusts. The general semi-infinite optimal control problem can be written

as:

$$
\begin{aligned}
& \min _{\Delta \tilde{V}} \sum_{i=1}^{N}\left\|\Delta \tilde{V}_{i}\right\|_{1} \\
& \text { s.t. }\left\{\begin{array}{l}
\left\|\Delta \tilde{V}_{i}\right\| \leq \overline{\Delta \tilde{V}_{i}}, \forall i=1 \ldots N \\
\tilde{X}\left(\nu_{1}\right)=\tilde{X}_{1} \\
M\left(\nu_{N}\right) \tilde{X}\left(\nu_{N}\right)=0 \\
(\tilde{x}(\nu), \tilde{y}(\nu), \tilde{z}(\nu)) \in \tilde{R}_{t o l}, \forall \nu \geq \nu_{N}
\end{array}\right.
\end{aligned}
$$

The state at the end of the maneuvers plan, $\tilde{X}\left(\nu_{N}\right)$, can be expressed as a function of the initial state $\tilde{X}\left(\nu_{1}\right)$ and the impulsive controls $\Delta \tilde{V}$ by using the Yamanaka-Ankersen transition matrix $\Phi$ :

$$
\tilde{X}\left(\nu_{N}\right)=\mathbf{A}_{N} \Delta \tilde{V}^{N}+\mathbf{B}_{N}
$$

where:

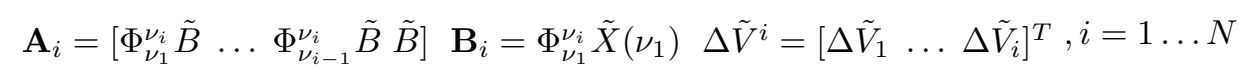

For convenience we choose the tolerance region $R_{t o l}$ to be a box centred around a desired position $X_{f}=\left[\begin{array}{lll}x_{f} & y_{f} & z_{f}\end{array}\right]^{T}$, whose dimensions are defined by $X_{t o l}=\left[\begin{array}{lll}x_{t o l} & y_{t o l} & z_{t o l}\end{array}\right]^{T}$. The tolerance box constraints can be easily written in the general linear form (30) where the $V$ and $K$ matrices are given by:

$$
V=\left[\begin{array}{ccc}
1 & 0 & 0 \\
-1 & 0 & 0 \\
0 & 1 & 0 \\
0 & -1 & 0 \\
0 & 0 & 1 \\
0 & 0 & -1
\end{array}\right], \quad K=\left[\begin{array}{c}
x_{f}-x_{t o l} \\
-x_{f}+x_{t o l} \\
y_{f}-y_{t o l} \\
-y_{f}+y_{t o l} \\
z_{f}-z_{t o l} \\
-z_{f}+z_{t o l}
\end{array}\right], \quad\left[\nu_{0}, \nu_{f}\right]=\left[\nu_{N}, \nu_{N}+2 \pi\right]
$$

Following the previously developed procedure, the infinitely many tolerance box constraints can be transformed into a finite number of non negativity constraints on some polynomials whose coefficients depend linearly on the decision variables $\Delta \tilde{V}$ :

$$
\Gamma_{i}(w)=T(w) k_{i}-v_{i, 1} P_{x p}(w)-v_{i, 2} \bar{P}_{y}(w)-v_{i, 3} P_{z p}(w) \geq 0, \forall w \in \mathbb{R}, i=1 \ldots 6
$$


The coefficients $\gamma_{i}$ of the polynomials $\Gamma_{i}(w)$ depend linearly on the state at the end of the maneuvres plan:

$$
\gamma_{i}=t k_{i}-\left(v_{i, 1} C_{x}+v_{i, 2} \bar{C}_{y}+v_{i, 3} C_{z}\right)\left(\mathbf{A}_{N} \Delta \tilde{V}^{N}+\mathbf{B}_{N}\right)
$$

Thus, using the previously developed results, the problem of finding the impulsive controls $\Delta V_{i}$ such that the chaser satellite reaches at the end of the prediction horizon a trajectory that is periodic and contained in a specified tolerance region is transformed into a semi-definite program:

$$
\begin{aligned}
& \min _{\Delta \tilde{V}, Z} \begin{array}{l}
\sum_{i=1}^{N} Z_{i} \\
-Z_{i} \leq \Delta \tilde{V}_{i} \leq Z_{i} \\
Z_{i} \leq \overline{\Delta \tilde{V}_{i}} \\
\tilde{X}\left(\nu_{1}\right)=\tilde{X}_{1} \\
M\left(\nu_{N}\right) \tilde{X}\left(\nu_{N}\right)=0 \\
\exists Y_{i} \succeq 0 \text { s.t. } \gamma_{i}=\Lambda^{*}\left(Y_{i}\right), \forall i=1 \ldots N
\end{array}
\end{aligned}
$$

To illustrate this particular guidance problem, we use the rendezvous mission summarized in table 1. The purpose is to reach a periodic parking relative orbit by applying the computed impulsive maneuvers. Our method for imposing continuous constraints on the resulting periodic trajectory is compared with a method based on constraints discretization [5]. Yalmip [27] along with the SDPT3 solver [28] is for solving the semi-definite programming (SDP) problem (54). The linear program (LP) corresponding to the method used for comparison is solved with the linprog function from Matlab.

Table 1: Simulation data for constrained periodic relative motion

\begin{tabular}{ccccccccc}
\hline \hline$e$ & $a[\mathrm{~km}]$ & $N$ & $X_{1}[\mathrm{~m}, \mathrm{~m} / \mathrm{s}]$ & $t_{1}[\mathrm{~s}]$ & $X_{f}[\mathrm{~m}]$ & $X_{\text {tol }}[\mathrm{m}]$ & $t_{N}[\mathrm{~s}]$ & $\overline{\Delta V}[\mathrm{~m} / \mathrm{s}]$ \\
\hline 0.023776 & \multirow{2}{*}{7011} & 10 & {$[1000,50,50,0,0,0]$} & 1282 & {$[100,0,0]$} & {$[20,10,10]$} & 18808 & 0.26 \\
\hline \hline
\end{tabular}

The obtained rendezvous trajectory is presented in figure 2. Both methods reach a periodic trajectory at the end of the plan. The difference is that, for the discretization based method, the tolerance region constraints are sometimes violated between the verification points. No constraints 


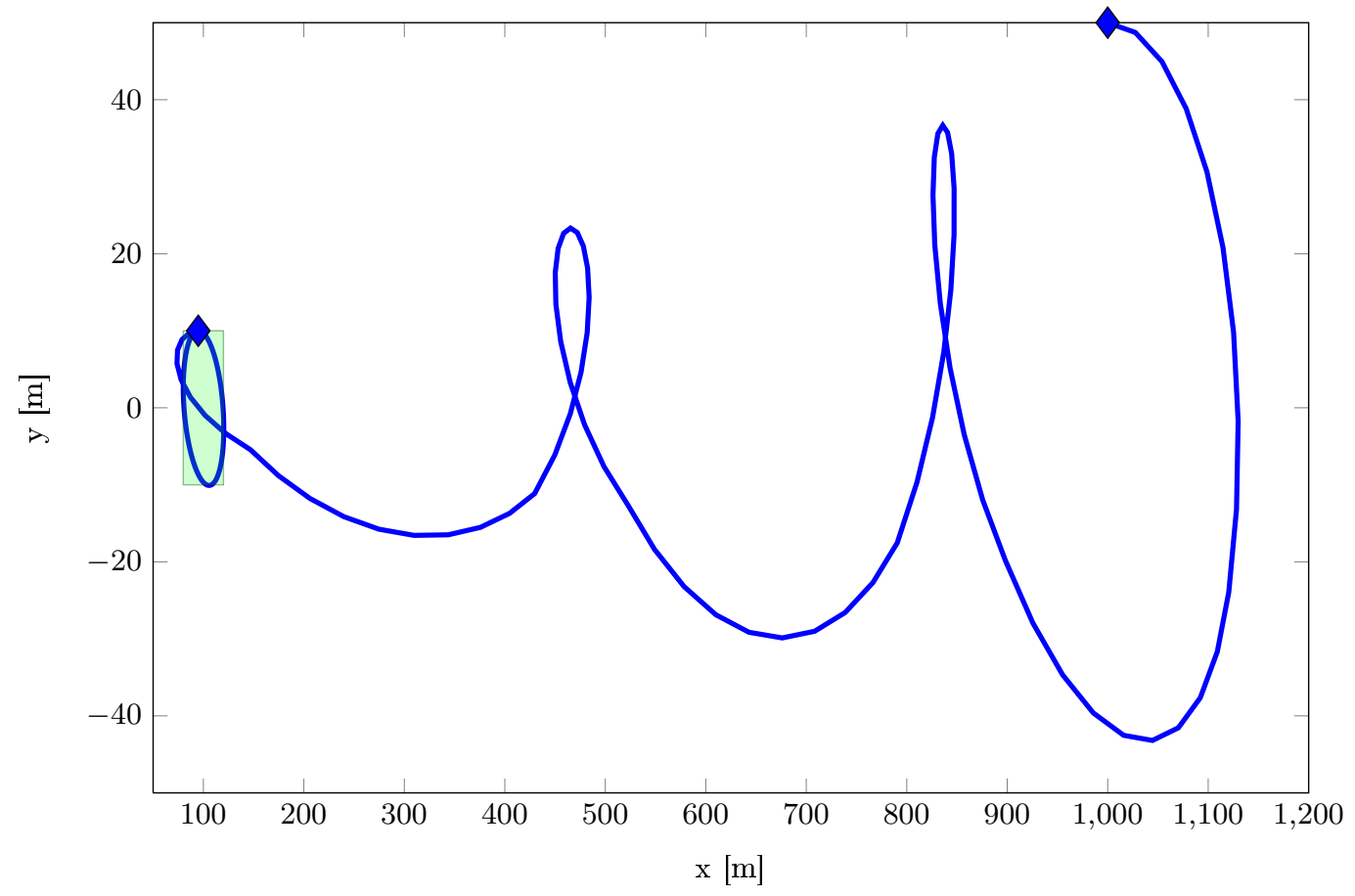

Fig. 2: The rendezvous trajectory

violations occur when using our method since it allows for the constraints on the relative trajectory to be imposed continuously in time.

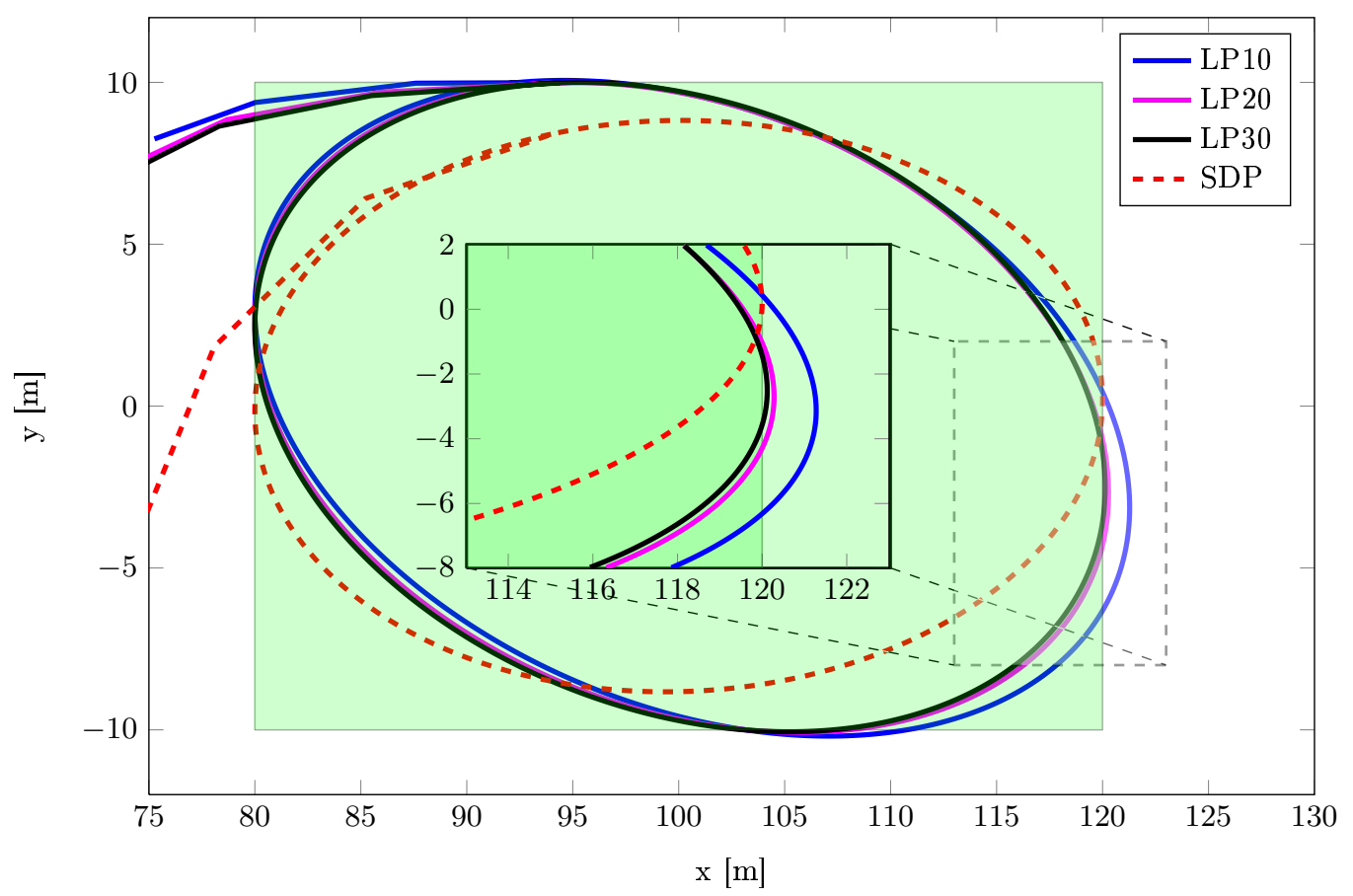

Fig. 3: The periodic trajectories obtained for each method 
For the discretization based method, the precision of the solution is influenced by the number of points where the constraints on the trajectory are explicitly checked. The resulting trajectories when considering 10, 20 and 30 points respectively are depicted in figure 3 . Table 2 shows the comparison between the fuel cost, the solver time and the time spent outside the tolerance region for each scenario. Increasing the number of verification points reduces the amount of constraints violations but it also increases the solver time and the fuel cost. For almost equal fuel cost, the SDP method has the advantage of guaranteeing zero constraints violation.

Table 2: Comparison between the SDP and LP based methods

\begin{tabular}{c|cccc}
\hline \hline Method & LP10 & LP20 & LP30 & SDP \\
\hline Fuel cost $[\mathrm{m} / \mathrm{s}]$ & 0.48907 & 0.48922 & 0.48927 & 0.48927 \\
Solver time $[\mathrm{s}]$ & 0.1972 & 0.6499 & 1.6241 & 0.9325 \\
Time out of bounds $[\mathrm{s}]$ & 1269 & 737 & 339 & 0 \\
\hline \hline
\end{tabular}

\section{B. Orbital rendezvous under passive safety constraints}

An important issue for the guidance algorithms for spacecraft proximity operations like orbital rendezvous and docking is the ability to handle abnormal system behaviour. The purpose is to ensure a safe behaviour for a large class of possible malfunctions. Passive safety implies the design of rendezvous trajectories such that disabling the follower's thrusters in the event of a failure will cause the satellites to remain on a relative fail trajectory that is guaranteed to be collision free [26]. Security constraints must be imposed both on the rendezvous trajectory and the predicted fail trajectories in order to guarantee this kind of behaviour.

The passive safety constraints can be imposed on any of the $N$ steps of the rendezvous plan but adding too many constraints will increase the total fuel cost of the mission without further improving the overall probability of collision [7]. That is why the security constraints will only be enforced on the last $S$ steps of the plan. In order to guarantee that the fail trajectories $\tilde{X}_{\text {fail }}$ are collision free, they are designed to be periodic and to evolve inside a specified area in proximity of the target $\mathcal{X}_{\text {safe }}$. This has to be achieved while minimizing the overall fuel cost of the mission and 

control problem can be written as:

$$
\begin{aligned}
& \min _{\Delta \tilde{V}} \sum_{i=1}^{N}\left\|\Delta \tilde{V}_{i}\right\|_{1} \\
& \text { s.t. }\left\{\begin{array}{l}
\left\|\Delta \tilde{V}_{i}\right\| \leq \overline{\Delta \tilde{V}_{i}}, \forall i=1 \ldots N \\
\tilde{X}\left(\nu_{1}\right)=\tilde{X}_{1} \\
M\left(\nu_{i}\right) \tilde{X}\left(\nu_{i}\right)=0 \\
\tilde{X}_{\text {fail }}^{i}(\nu) \in \mathcal{X}_{\text {safe }}, \forall \nu \geq \nu_{i} \\
\tilde{X}_{f}-\tilde{X}_{t o l} \leq \tilde{X}\left(\nu_{N}\right) \leq \tilde{X}_{f}+\tilde{X}_{t o l}
\end{array}\right.
\end{aligned}
$$

The fail trajectories $\tilde{X}_{\text {fail }}$ are obtained through the open loop propagation of the spacecraft autonomous relative motion starting from the states on the rendezvous trajectory that must be rendered passively safe. The safe area $\mathcal{X}_{\text {safe }}$ is considered to be an open polytope behind the chaser defined by :

$$
\tilde{x}_{\text {fail }}^{i}(\nu) \leq \tilde{x}_{\text {safe }}, \quad \forall \nu \geq \nu_{i}, \quad \forall i=N-S \ldots N-1
$$

Following the same procedure as before, constraints (56) can be written as:

$$
\Gamma_{i}(w) \geq 0, \quad \forall w \in \mathbb{R}
$$

with:

$$
\Gamma_{i}(w)=T(w) x_{\mathrm{safe}}-P_{x p}^{i}(w), \quad \forall i=N-S \ldots N-1
$$

where $P_{x p}^{i}(w)$ is the polynomial for the propagation of the autonomous periodic motion on the $x$ axis starting from each state $\tilde{X}\left(\nu_{i}\right)$. In addition, the state $\tilde{X}\left(\nu_{i}\right)$ must verify the periodicity condition (23). The coefficients $\gamma_{i}$ of the polynomials $\Gamma_{i}(w)$ can be written directly as a linear function of the decision variables $\Delta \tilde{V}_{i}$ :

$$
\gamma_{i}=-C_{x} C_{p}\left(\nu_{i}\right)\left(\mathbf{A}_{i} \Delta \tilde{V}^{i}+\mathbf{B}_{i}\right)+x_{\text {safe }} t, \quad \forall i=N-S \ldots N-1
$$

Using the LMI conditions (46), the semi-infinite optimization problem (55) can be transformed into 
a semi-definite program:

$$
\begin{aligned}
& \min _{\Delta \tilde{V}, Z} \sum_{i=1}^{N} Z_{i} \\
& \text { s.t. }\left\{\begin{array}{l}
-Z_{i} \leq \Delta \tilde{V}_{i} \leq Z_{i} \quad, \forall i=1 \ldots N \\
Z_{i} \leq \overline{\Delta \tilde{V}_{i}} \quad\left\{\begin{array}{l}
\tilde{X}\left(\nu_{1}\right)=\tilde{X}_{1} \\
M\left(\nu_{i}\right)\left(\mathbf{A}_{i} \Delta \tilde{V}^{i}+\mathbf{B}_{i}\right)=0 \\
\exists Y_{i} \succeq 0 \text { s.t. }-C_{x} C_{p}\left(\nu_{i}\right)\left(\mathbf{A}_{i} \Delta \tilde{V}^{i}+\mathbf{B}_{i}\right)+x_{\mathrm{safe}} t=\Lambda^{*}\left(Y_{i}\right) \\
\tilde{X}_{f}-\tilde{X}_{t o l} \leq \mathbf{A}_{N} \Delta \tilde{V}^{N}+\mathbf{B}_{N} \leq \tilde{X}_{f}+\tilde{X}_{t o l}
\end{array}, \forall i=N-S \ldots N-1\right.
\end{array}\right.
\end{aligned}
$$

The data presented in table 3 corresponds to the rendezvous and docking mission with passive security constraints that is considered for illustration.

In order to identify a suitable value for the security horizon $S$, the rendezvous mission without

Table 3: Simulation data for the rendezvous mission with passive security constraints

\begin{tabular}{ccccccccc}
\hline \hline$e$ & $a[\mathrm{~km}]$ & $N$ & $X_{1}[\mathrm{~m}, \mathrm{~m} / \mathrm{s}]$ & $t_{1}[\mathrm{~s}]$ & $X_{f}[\mathrm{~m}, \mathrm{~m} / \mathrm{s}]$ & $v_{t o l}[\mathrm{~m} / \mathrm{s}]$ & $t_{N}[\mathrm{~s}]$ & $x_{\text {safe }}[\mathrm{m}]$ \\
\hline 0.023776 & 7011 & 15 & {$[-30,0,-3,0,0,0]$} & 0 & {$[-5,0,0,0,0,0]$} & 0.01 & 5843 & -5 \\
\hline \hline
\end{tabular}

security constraints is solved first. The fail trajectories are propagated starting from every controlled state on the second half of the rendezvous plan. The resulting trajectories are presented in figure 4. The states which originate fail trajectories leading to a collision between the two satellites are then included in the safety horizon $S$. Figure 4 suggests that a security horizon of $S=4$ should remove the collision risk in case of system failure (please note that some fail trajectories overlap for the states where the optimized $\Delta V$ equals zero).

Figure 5 presents the fail trajectories obtained when the passive security constraints are enforced in the control synthesis problem for a security horizon of $S=4$. The fail trajectories are indeed periodic and evolve in the security area defined by $x_{\text {safe }}$, removing the risk of collision in case of system error. 


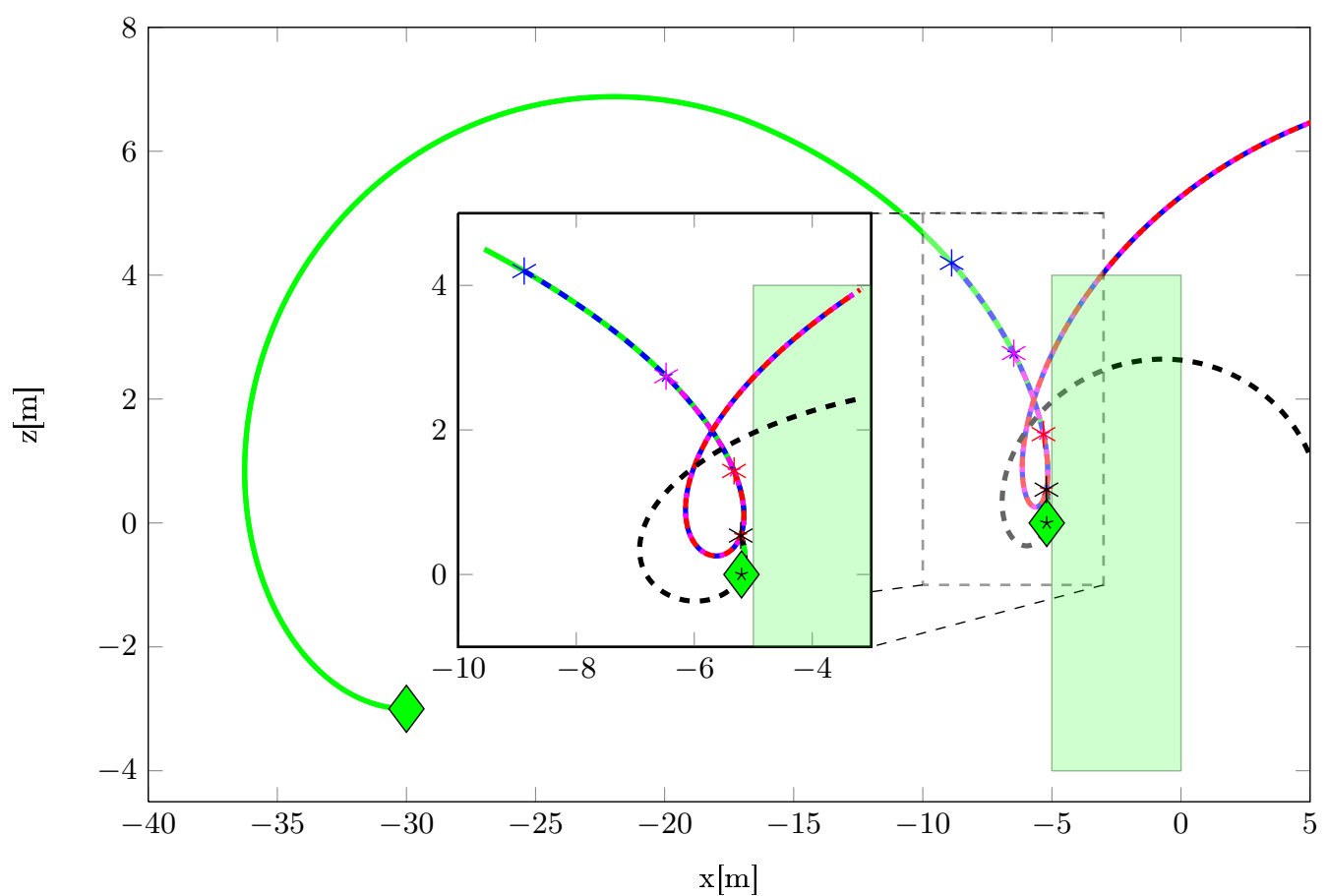

Fig. 4: The rendezvous trajectory without the security constraints (the dashed lines represent the fail trajectories starting from the points evidenced by the ${ }^{*}$ symbol)

Table 4 shows the evolution of the mission fuel cost with respect to the choice of the security horizon $S$. As expected the fuel cost increases as the security horizon increases since more and more constraints are added to the problem.

Table 4: Evolution of the mission fuel cost with the length of the security horizon

\begin{tabular}{c|cccccccc}
\hline \hline$S$ & 0 & 1 & 2 & 3 & 4 & 5 & 6 & 7 \\
\hline Fuel cost $[\mathrm{m} / \mathrm{s}]$ & 0.0116 & 0.0121 & 0.0135 & 0.0146 & 0.0156 & 0.0163 & 0.0168 & 0.0174 \\
\hline \hline
\end{tabular}

The security constraints considered in this example were very simple in order to highlight the principle of the method without too much formal complexity. The presented method can easily accommodate different descriptions of the safe region, like for instance a visibility cone as in [7].

\section{Orbital rendezvous under visibility constraints}

The previous examples featured scenarios where the constraints are imposed on periodic relative trajectories. A rendezvous mission with visibility constraints is now considered in order to illustrate 


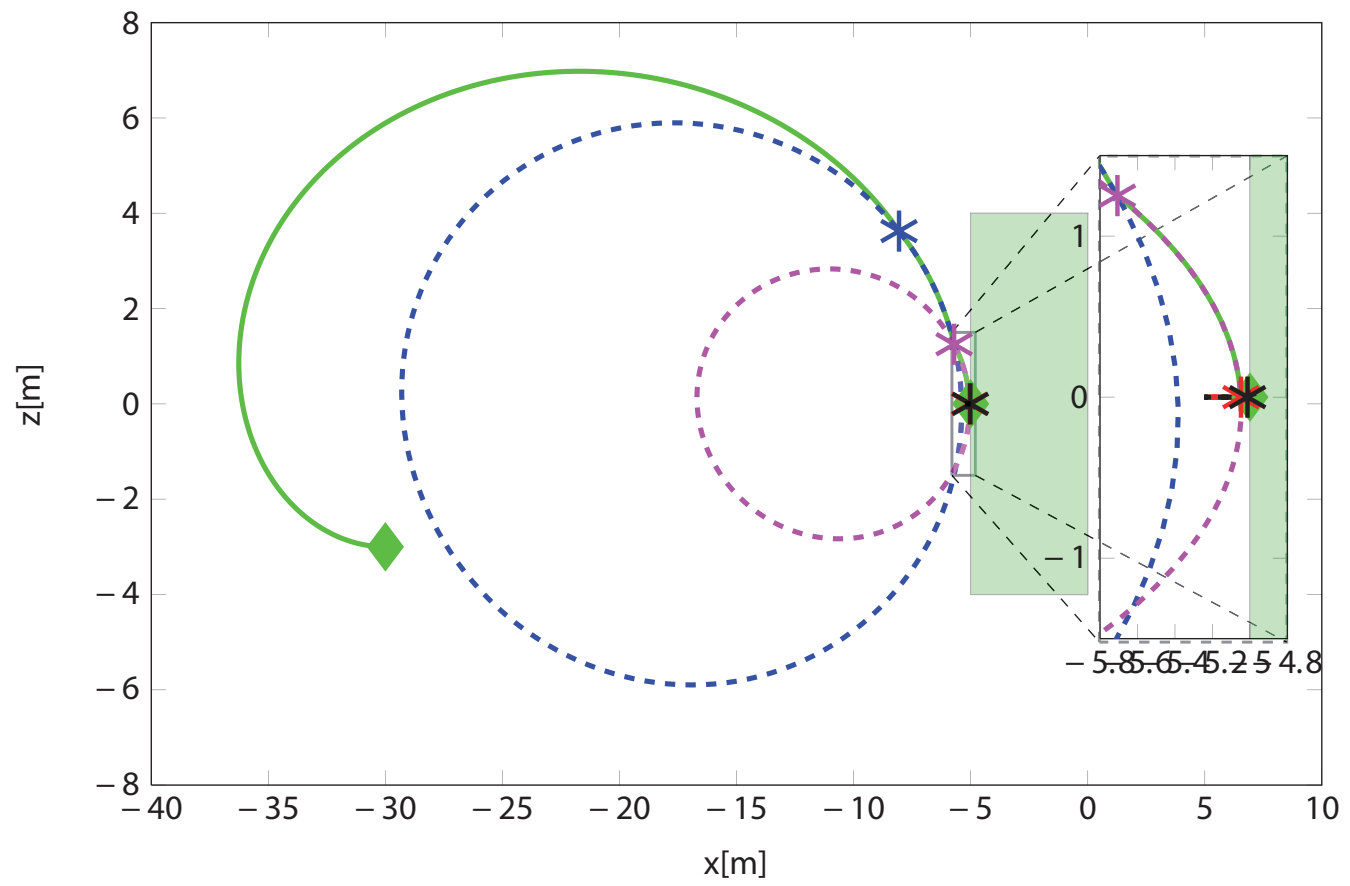

Fig. 5: The rendezvous trajectory with security constraints (the dashed lines represent the fail trajectories starting from the points evidenced by the ${ }^{*}$ symbol)

the use of the non negative polynomials in imposing continuous constraints on non periodic spacecraft relative trajectories. The chosen mission requires for the follower to remain inside the leader's visibility cone for the entire duration of the rendezvous and docking maneuvers. The optimal control problem can be formulated as follows:

$$
\begin{array}{ll}
\min _{\Delta \tilde{V}} & \sum_{i=1}^{N}\left\|\Delta \tilde{V}_{i}\right\|_{1} \\
\text { s.t. } & \left\{\begin{array}{l}
\left\|\Delta \tilde{V}_{i}\right\| \leq \overline{\Delta \tilde{V}_{i}}, \forall i=1 \ldots N \\
\tilde{X}\left(\nu_{1}\right)=\tilde{X}_{1} \\
(\tilde{x}(\nu), \tilde{y}(\nu), \tilde{z}(\nu)) \in \mathcal{X}_{\text {vis }}, \forall \nu \in\left[\nu_{1}, \nu_{N}\right] \\
\tilde{X}\left(\nu_{N}\right)=\tilde{X}_{f}
\end{array}\right.
\end{array}
$$


Using the previously developed method, continuous satisfaction of the visibility constraints can be guaranteed for each time segment between two thrust positions $\left[\nu_{i}, \nu_{i+1}\right]$ :

$$
\begin{aligned}
& \min _{\Delta \tilde{V}} \sum_{i=1}^{N}\left\|\Delta \tilde{V}_{i}\right\|_{1} \\
& \text { s.t. }\left\{\begin{array}{l}
\left\|\Delta \tilde{V}_{i}\right\| \leq \overline{\Delta \tilde{V}_{i}}, \forall i=1 \ldots N \\
(\tilde{x}(\nu), \tilde{y}(\nu), \tilde{z}(\nu)) \in \mathcal{X}_{\mathrm{vis}}, \forall \nu \in\left[\nu_{1}, \nu_{2}\right] \\
(\tilde{x}(\nu), \tilde{y}(\nu), \tilde{z}(\nu)) \in \mathcal{X}_{\mathrm{vis}}, \forall \nu \in\left[\nu_{2}, \nu_{3}\right] \\
\vdots \\
(\tilde{x}(\nu), \tilde{y}(\nu), \tilde{z}(\nu)) \in \mathcal{X}_{\mathrm{vis}}, \forall \nu \in\left[\nu_{N-1}, \nu_{N}\right] \\
\tilde{X}\left(\nu_{N}\right)=\tilde{X}_{f}
\end{array}\right.
\end{aligned}
$$

The set $\mathcal{X}_{\mathrm{vis}}$ is associated to the visibility cone of the leader's camera. It is represented by an open polytope behind the leader satellite (figure 6), defined by the aperture angle $\beta$ and the offset distance $x_{\text {safe }}$ between the docking port of the leader satellite and its center of gravity.

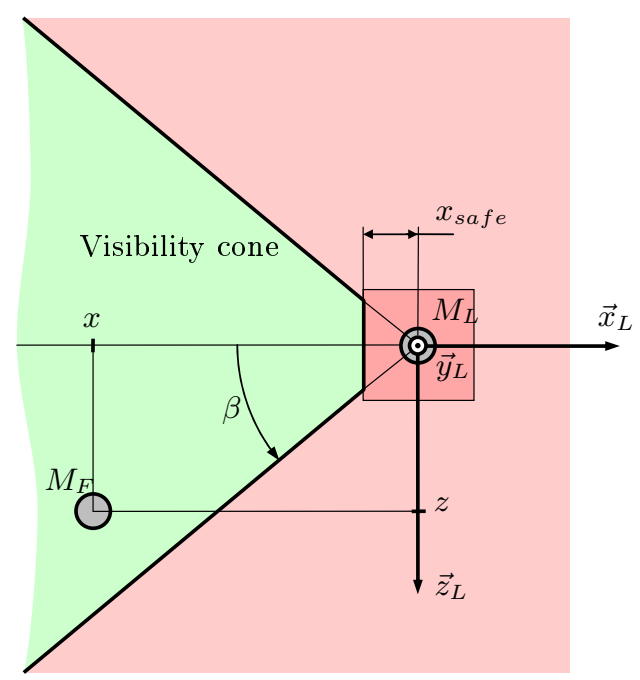

Fig. 6: The leader spacecraft visibility cone

The visibility constraints can easily be written as a linear inequality like in (30) with the $V$ and 
$K$ matrices defined by:

$$
V=\left[\begin{array}{ccc}
1 & 0 & \rho \\
1 & 0 & -\rho \\
1 & \rho & 0 \\
1 & -\rho & 0 \\
1 & 0 & 0
\end{array}\right] \quad K=\left[\begin{array}{c}
0 \\
0 \\
0 \\
0 \\
x_{\mathrm{safe}}
\end{array}\right]
$$

and $\rho=\tan \left(\frac{\pi}{2}-\beta\right)$.

Expanding the visibility cone constraints leads to polynomial non negativity constraints similar to (36). For the sake of completeness, the procedure for the first visibility constraint is fully detailed:

$$
-\tilde{x}(\nu)-\rho \tilde{z}(\nu) \geq 0, \forall \nu \in\left[\nu_{i}, \nu_{i+1}\right], \forall i=1 \ldots N-1
$$

Using the variable change (12) and the expressions (13) for the propagation of the relative motion starting from each controlled state $\tilde{X}\left(\nu_{i}\right)$ on the rendezvous trajectory, the first visibility constraint can further be transformed in:

$$
\Gamma_{1}^{i}(w) \geq 0, \forall w \in\left[\begin{array}{ll}
w_{i} & w_{i+1}
\end{array}\right], \forall i=1 \ldots N-1
$$

where:

$\Gamma_{1}^{i}(w)=-\left[P_{x}^{i}(w)+3 d_{4}^{i} P_{J x}(w) J_{\nu}^{i}(w)\right]-\rho\left[P_{z}^{i}(w)+2 d_{4}^{i}\left(P_{J z}(w) J_{\nu}^{i}\right)\right] \geq 0, \forall w \in\left[w_{i}, w_{i+1}\right], \forall i=1 \ldots N-1$

The term $J_{\nu}^{i}(w)$ in (65) is replaced on each segment $\left[w_{i}, w_{i+1}\right]$ by its upper and its lower polynomial bounds, $\Theta_{u}^{i}(w)$ and $\Theta_{l}^{i}(w)$ respectively. This leads to the following polynomial non negativity constraints:

$$
\begin{aligned}
& \Gamma_{1 l}^{i}(w)=-\left[P_{x}^{i}(w)+\rho P_{z}^{i}(w)\right]-d_{4}\left[3 P_{J x}(w)+2 \rho P_{J z}\right] \Theta_{l}^{i}(w) \geq 0 \\
& \Gamma_{1 u}^{i}(w)=-\left[P_{x}^{i}(w)+\rho P_{z}^{i}(w)\right]-d_{4}\left[3 P_{J x}(w)+2 \rho P_{J z}\right] \Theta_{u}^{i}(w) \geq 0
\end{aligned}, \forall w \in\left[w_{i}, w_{i+1}\right], \forall i=1 \ldots N-1
$$

The degree of the polynomials $\Gamma_{l}^{i}(w)$ and $\Gamma_{u}^{i}(w)$ is $r+4$, where $r$ is the degree of the polynomial approximation for the drifting term $J_{\nu}^{i}(w)$ on each interval. The vectors of coefficients for $\Gamma_{l}^{i}(w)$ 
and $\Gamma_{u}^{i}(w)$, denoted by $\gamma_{l}^{i}(w)$ and $\gamma_{u}^{i}(w)$ respectively, depend linearly on the decision variables $\Delta \tilde{V}_{i}$ through the coefficients of the polynomials $P_{x}^{i}(w)$ and $P_{z}^{i}(w)$. We remind that the coefficients $p_{x}^{i}$ and $p_{z}^{i}$ of the polynomials $P_{x}^{i}(w)$ and $P_{z}^{i}(w)$ depend linearly on the relative state starting from which the satellites motion is propagated on the current time segment:

$$
\begin{aligned}
& p_{x}^{i}=C_{x} C\left(\nu_{i}\right)\left(\mathbf{A}_{i} \Delta \tilde{V}^{i}+\mathbf{B}_{i}\right) \\
& p_{z}^{i}=C_{z} C\left(\nu_{i}\right)\left(\mathbf{A}_{i} \Delta \tilde{V}^{i}+\mathbf{B}_{i}\right)
\end{aligned}
$$

where $\mathbf{A}_{i}, \mathbf{B}_{i}$ and $\Delta \tilde{V}^{i}$ are defined in (50). This dependence implies that a wise choice of the impulsive controls $\Delta \tilde{V}_{i}$ can guarantee the continuous satisfaction of the visibility constraints all along the rendezvous path. As seen in the previous section, constraints (66) are satisfied if and only if:

$$
\begin{aligned}
& \exists\left(Y_{1 l}^{i} \succeq 0, Y_{2 l}^{i} \succeq 0\right) \text { s.t. } \gamma_{l}^{i}=\Lambda^{*}\left(Y_{1 l}^{i}, Y_{2 l}^{i}\right) \\
& \exists\left(Y_{1 u}^{i} \succeq 0, Y_{2 u}^{i} \succeq 0\right) \text { s.t. } \gamma_{u}^{i}=\Lambda^{*}\left(Y_{1 u}^{i}, Y_{2 u}^{i}\right)
\end{aligned}
$$

The constrained rendezvous problem (61) is finally written as the concatenation of the LMI conditions $(67)$, for each visibility constraint and for each time segment $\left[w_{i}, w_{i+1}\right]$ :

$$
\begin{aligned}
& \min _{\Delta \tilde{V}, Z} \sum_{i=1}^{N} Z_{i} \\
& \text { s.t. } \begin{cases}-Z_{i} \leq \Delta \tilde{V}_{i} \leq Z_{i}, & \forall i=1 \ldots N \\
Z_{i} \leq \overline{\Delta \tilde{V}_{i}} & \\
\gamma_{j l}^{i}=\Lambda^{*}\left(Y_{j 1 l}^{i}, Y_{j 2 l}^{i}\right), Y_{j 1 l}^{i} \succeq 0, Y_{j 2 l}^{i} \succeq 0 \quad & , \quad \forall i=1, \ldots, N-1, \forall j=1, \ldots, 5 \\
\gamma_{j u}^{i}=\Lambda^{*}\left(Y_{j 1 u}^{i}, Y_{j 2 u}^{i}\right), Y_{j 1 u}^{i} \succeq 0, Y_{j 2 u}^{i} \succeq 0 & \\
\tilde{X}\left(\nu_{N}\right)=\tilde{X}_{f} & \end{cases}
\end{aligned}
$$

where the superscript $i$ identifies the time segment and the subscript $j$ identifies the index of the visibility constraint in (62).

The rendezvous mission summarized in table 5 is used to illustrate this approach. The initial position for the rendezvous is $\nu_{1}=-\pi / 2$, chosen negative so that the maneuvers start before the current passage through the perigee. This choice is motivated by the fact that the values of $w$ must be strictly increasing on each interval $\left[w_{i}, w_{i+1}\right]$ in order to obtain accurate polynomial 
approximations of the drifting term $J(w)$. The variable change (12) maps the interval of one orbital period $[-\pi, \pi]$ to $\mathbb{R}$ and the duration for the rendezvous is chosen to be half of an orbital period in order to get an interval where the polynomial approximations are more precise. A tolerance of 0.001 $\mathrm{m} / \mathrm{s}$ is allowed on the final speed and the visibility cone is defined by the parameters $x_{\text {safe }}=-5[\mathrm{~m}]$ and $\beta=20^{\circ}$.

Table 5: Simulation data for the rendezvous mission with visibility constraints

\begin{tabular}{cccccccc}
\hline \hline$e$ & $a[\mathrm{~km}]$ & $N$ & $\nu_{1}[\mathrm{rad}]$ & $X_{1}[\mathrm{~m}, \mathrm{~m} / \mathrm{s}]$ & $\nu_{N}[\mathrm{rad}]$ & $X_{f}[\mathrm{~m}, \mathrm{~m} / \mathrm{s}]$ & $\overline{\Delta V}[\mathrm{~m} / \mathrm{s}]$ \\
\hline 0.023776 & 7011 & 5 & $-\pi / 2$ & {$[-50 ;-10 ; 15 ; 0 ; 0 ; 0]^{T}$} & $\pi / 2$ & {$[-6 ; 0 ; 0 ; 0 ; 0 ; 0]^{T}$} & 0.26 \\
\hline \hline
\end{tabular}

The software Sollya [29] is used for obtaining the polynomial approximations for the term $J(w)$ on each segment of the rendezvous plan. The degree of the approximations is fixed to $r=2$. The maximal certified approximation error $\varepsilon$ is about $0.25 \%$ (figures 7 ).

Time segment $1 \quad$ Time segment $2 \quad$ Time segment 3

Time segment 4
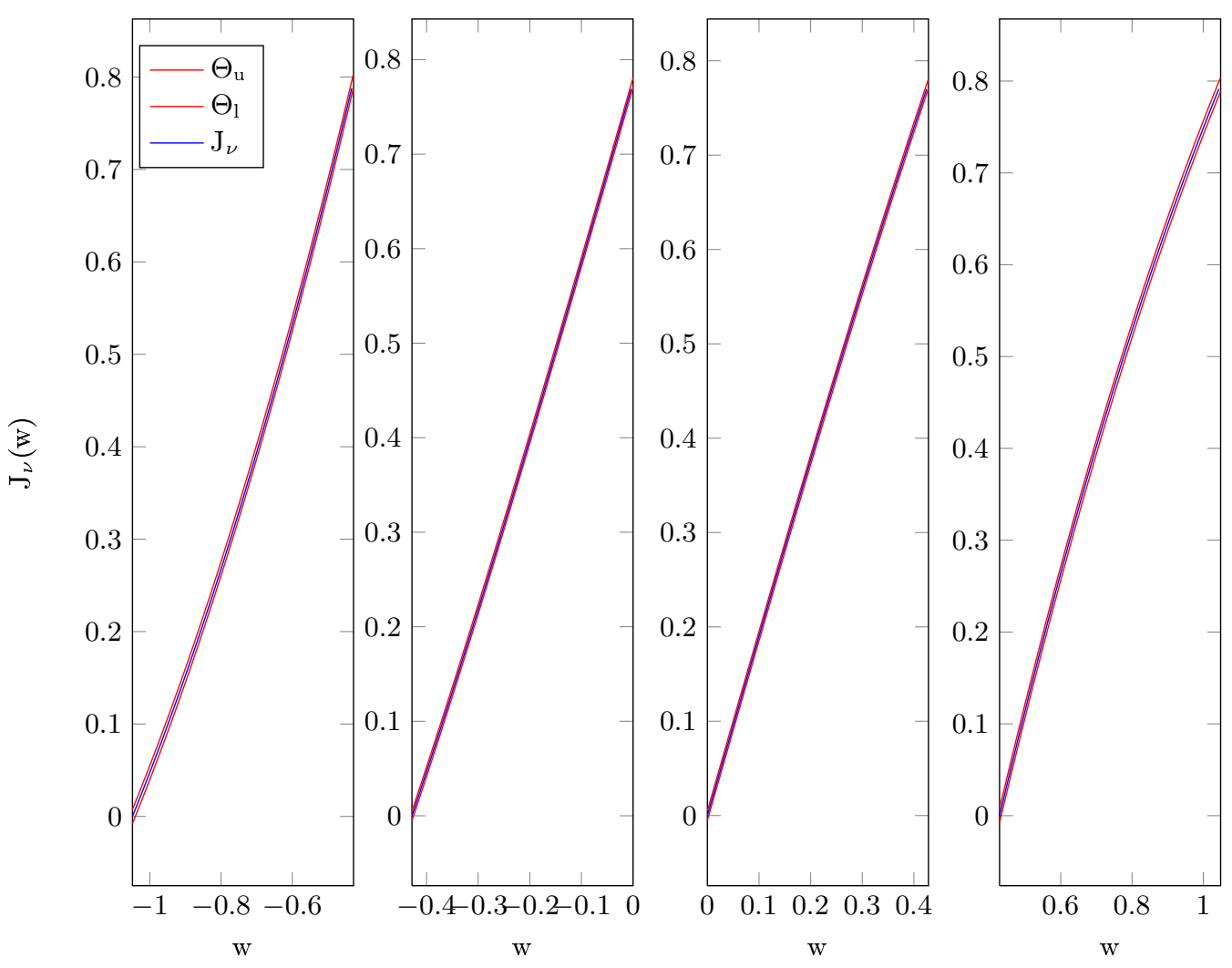

Fig. 7: Drifting term $J(w)$ and its approximation on each time segment 
The solution of the final semi-definite programming problem is obtained using Yalmip [27] and the SDPT3 [28] solver. A method based on constraints discretization is used for comparison [5]. The discretized problem amounts to a linear program (LP) whose solution is obtained using the linprog function from Matlab.

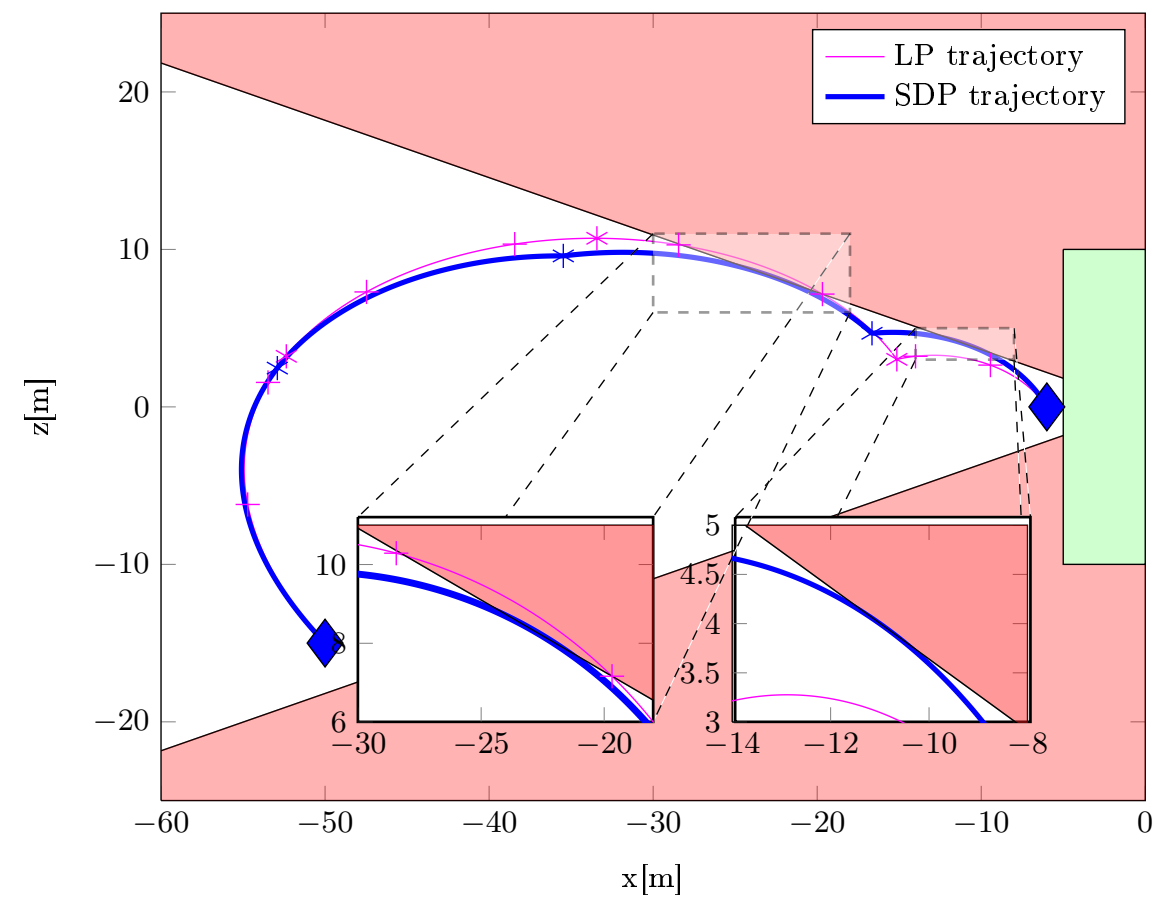

Fig. 8: Comparison between the $x z$ projections of the rendezvous trajectories

Figures 8 and 9 present the comparison between the trajectories obtained with each method, where 10 collocation points are used for constraints discretization. It can be seen that the trajectory obtained for the discertized problem sometimes goes outside from the visibility cone in between the points where the constraints are explicitly checked. Unlike our method, the constraints discretization based method cannot guarantee continuous satisfaction of the constraints even if the amount of constraints violation may be reduced by increasing the number of collocation points (table 6). The obtained solution, even if slightly less fuel expensive, is only optimal for the discrete problem.

It is worth noting that when the number of collocation points is increased, the $\Delta V$ cost issued by the LP problem tends towards the value given by the SDP problem. This suggests that the LP problem is a relaxation of the SDP problem (68) through discretization.

Both the LP and the SDP problems can be solved using interior point algorithms. The resolution 
constraints discretization. Even if effective, the discretization may lead to constraints violations in between the verification points. Using a rational parametrization of the linearised spacecraft relative motion, we are able to transform the infinitely many constraints on the trajectory into a finite number of polynomial non negativity constraints. This formulation provides a priori guarantees on the obtained solution. The optimization problem is finally formulated as a semi-definite program that can be effectively solved using interior point methods.

The efficiency of the method is demonstrated using several spacecraft mission scenarios corresponding to different types of proximity operations.

\section{Acknowledgments}

This work was supported by the French National Center for Space Studies (CNES) and by Astrium-EADS.

\section{References}

[1] Saleh, J., Hastings, D., Lamassoure, E., and Newman, D., "Flexibility and the Value of On-Orbit Servicing: New Customer-Centric Perspective," Journal of Spacecraft and Rockets, Vol. 40, No. 2, 2003, pp. 279-291.

[2] Breger, L., How, J., and Richards, A., "Model predictive control of spacecraft formations with sensing noise," American Control Conference (ACC), 2005, pp. 2385-2390.

[3] Mueller, J. and Larsson, R., "Collision avoidance maneuver planning with robust optimization," 7th International ESA Conference on Guidance, Navigation and Control Systems, No. June, 2008.

[4] Louembet, C. and Deaconu, G., "Collision avoidance in low thrust rendezvous guidance using flatness and positive B-splines," American Control Conference (ACC), 2011.

[5] Tillerson, M., Inalhan, G., and How, J. P., "Co-ordination and control of distributed spacecraft systems using convex optimization techniques," International Journal of Robust and Nonlinear Control, Vol. 12, No. 2-3, 2002, pp. 207-242.

[6] Larsson, R., Berge, S., Bodin, P., and Jonsson, U., "Fuel Efficient Relative Orbit Control Strategies for Formation Flying and Rendezvous within PRISMA," 29th AAS Guidance and Control Conference, 2006.

[7] Breger, L. and How, J., "Safe Trajectories for Autonomous Rendezvous of Spacecraft," Journal of Guidance, Control, and Dynamics, Vol. 31, No. 5, 2008, pp. 1478-1489. 
[8] Park, H., Di Cairano, S., and Kolmanovsky, I., "Model Predictive Control of spacecraft docking with a non-rotating platform," 18th IFAC World Congress, 2011.

[9] Irvin, D., Cobb, R., and Lovell, T., "Fuel-Optimal Maneuvers for Constrained Relative Satellite Orbits," Journal of Guidance, Control, and Dynamics, Vol. 32, No. 3, 2009, pp. 960-973.

[10] Williams, J. and Lightsey, E., "Optimal impulsive maneuvering within a confined hover region," $A I A A$ Guidance, Navigation and Control Conference and Exhibit, 2008.

[11] Hartley, E., Trodden, P., Richards, A., and Maciejowski, J., "Model predictive control system design and implementation for spacecraft rendezvous," Control Engineering Practice, Vol. 20, No. 7, 2012, pp. 695-713.

[12] Tschauner, J., "Elliptic orbit rendezvous," AIAA Journal, Vol. 5, No. 6, 1967, pp. 1110-1113.

[13] Yamanaka, K. and Ankersen, F., "New state transition matrix for relative motion on an arbitrary elliptical orbit," Journal of Guidance, Control, and Dynamics, Vol. 25, No. 1, 2002, pp. 60-66.

[14] Alfriend, K. and Schaub, H., "Dynamics and control of spacecraft formations- Challenges and some solutions," Journal of the Astronautical Sciences, Vol. 48, No. 2, 2000, pp. 249-267.

[15] Gurfil, P., "Relative Motion between Elliptic Orbits: Generalized Boundedness Conditions and Optimal Formationkeeping," Journal of Guidance, Control, and Dynamics, Vol. 28, No. 4, 2005, pp. 761-767.

[16] Gurfil, P. and Kholshevnikov, K., "Manifolds and Metrics in the Relative Spacecraft Motion Problem," Journal of Guidance, Control, and Dynamics, Vol. 29, No. 4, 2006, pp. 1004-1010.

[17] Sengupta, P. and Vadali, S., "Relative Motion and the Geometry of Formations in Keplerian Elliptic Orbits," Journal of Guidance, Control, and Dynamics, Vol. 30, No. 4, 2007, pp. 953-964.

[18] Jiang, F., Li, J., Baoyin, H., and Gao, Y., "Study on Relative Orbit Geometry of Spacecraft Formations in Elliptical Reference Orbits," Journal of Guidance, Control, and Dynamics, Vol. 31, No. 1, 2008, pp. $123-134$.

[19] Lane, C. and Axelrad, P., "Formation design in eccentric orbits using linearized equations of relative motion," Journal of Guidance, Control, and Dynamics, Vol. 29, No. 1, 2006, pp. 146-160.

[20] Breger, L., Inalhan, G., Tillerson, M., and How, J., "Cooperative Spacecraft Formation Flying: Model Predictive Control with Open- and Closed-Loop Robustness," Modern Astrodynamics, edited by P. Gurfil, chap. 8, Academic Press, 2007, pp. 237-277.

[21] Deaconu, G., Louembet, C., and Théron, A., "Constrained periodic spacecraft relative motion using non negative polynomials," American Control Conference (ACC), 2012.

[22] Chevillard, S., Harrison, J., Joldes, M., and Lauter, C., "Efficient and accurate computation of upper bounds of approximation errors," Theoretical Computer Science, Vol. 412, No. 16, 2011, pp. 1523-1543. 
[23] Nesterov, Y., "Squared functional systems and optimization problems," High performance optimization, chap. 17, Kluwer Academic Publishers, 2000.

[24] Ben-Tal, A. and Nemirovski, A., "Robust solutions of uncertain linear programs," Operations Research Letters, Vol. 25, No. 1, 1999, pp. 1-13.

[25] Deaconu, G., Louembet, C., and Théron, A., "A two-impulse method for stabilizing the spacecraft relative motion with respect to a periodic trajectory," Conference on Decision and Control (CDC), 2012.

[26] Fehse, W., Automated Rendezvous and Docking of Spacecraft, Cambridge University Press, 2003.

[27] Lofberg, J., "YALMIP: A toolbox for modeling and optimization in MATLAB," IEEE International Symposium on Computer Aided Control Systems Design, 2004, pp. 284-289.

[28] Toh, K., Tütüncü, R., and Todd, M., "SDPT3-a Matlab software package for semidefinite-quadraticlinear programming," Optimization Methods and Software, Vol. 11, 1999, pp. 545--581.

[29] Chevillard, S., Joldes, M., and Lauter, C., "Sollya: An environment for the development of numerical codes," Mathematical Software-ICMS, 2010, pp. 3-6. 